\title{
Seizures are a druggable mechanistic link between TBI and subsequent tauopathy
}

Hadeel Alyenbaawi $i^{1,2,7}$, Richard Kanyo ${ }^{1,3}$, Laszlo F. Locskai ${ }^{1,3}$, Razieh Kamali-Jamil ${ }^{1,4}$, Michèle G. DuVal ${ }^{3}$, Qing Bai ${ }^{5}$, Holger Wille ${ }^{1,4}$, Edward A. Burton ${ }^{5,6}$, W. Ted Allison ${ }^{1,2,3^{*}}$

${ }^{1}$ Centre for Prions \& Protein Folding Disease, University of Alberta, Edmonton AB, T6G 2M8, Canada

${ }^{2}$ Department of Medical Genetics, University of Alberta, Edmonton AB, T6G 2H7, Canada

${ }^{3}$ Department of Biological Sciences, University of Alberta, Edmonton AB, T6G 2E9, Canada

${ }^{4}$ Department of Biochemistry, University of Alberta, Edmonton, AB, Canada

${ }^{5}$ Department of Neurology, University of Pittsburgh, Pittsburgh, PA, USA

${ }^{6}$ Geriatric Research, Education and Clinical Center, Pittsburgh VA Healthcare System, Pittsburgh, PA, USA.

${ }^{7}$ Majmaah University, Majmaah,11952, Saudi Arabia

* Author for correspondence: ted.allison@ualberta.ca, 780-492-4430

Running title: Seizures link TBI to subsequent Tauopathy 


\section{Graphical Abstract:}

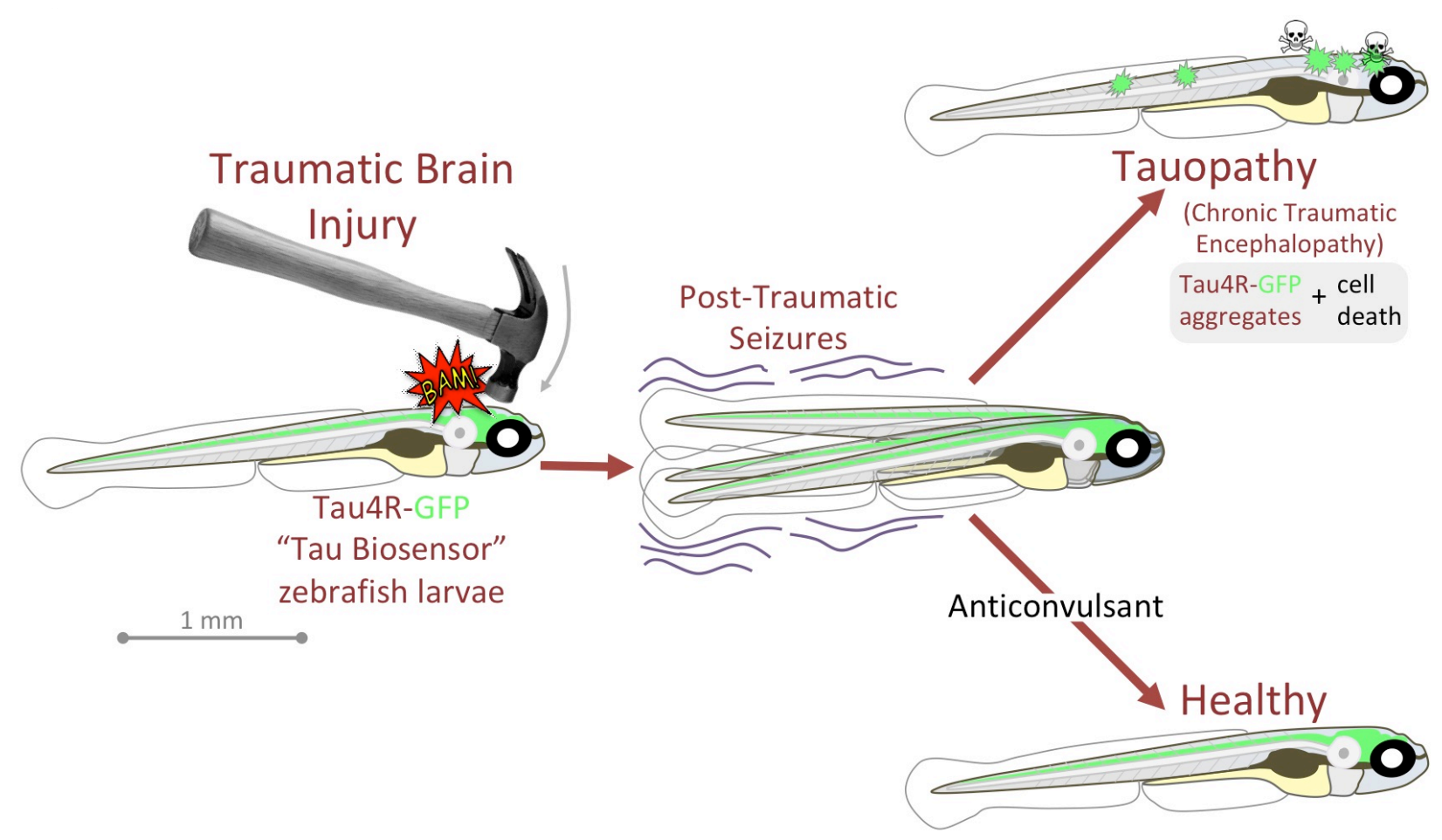

\section{Highlights:}

- Introduces first Traumatic Brain Injury (TBI) model in larval zebrafish, and its easy

- TBI induces clinically relevant cell death, haemorrhage \& post-traumatic seizures

- $\mathrm{Ca}^{2+}$ imaging during TBI reveals spike in brain activity concomitant with seizures

- Tau-GFP Biosensor allows repeated in vivo measures of prion-like tau aggregation

- post-TBI, anticonvulsants stop tauopathies akin to Chronic Traumatic Encephalopathy 


\section{Summary:}

1 Traumatic brain injury (TBI) is a prominent risk factor for neurodegenerative diseases

2 and dementias including chronic traumatic encephalopathy (CTE). TBI and CTE, like all

3 tauopathies, are characterized by accumulation of Tau into aggregates that

4 progressively spread to other brain regions in a prion-like manner. The mechanisms that

5 promote spreading and cellular uptake of tau seeds after TBI are not fully understood, in

6 part due to lack of tractable animal models. Here, we test the putative roles for excess

7 neuronal activity and dynamin-dependent endocytosis in promoting the in vivo spread of

8 tauopathy. We introduce 'tauopathy reporter' zebrafish expressing a genetically-

9 encoded fluorescent Tau biosensor that reliably reports accumulation of human tau

10 species when seeded via intra-ventricular brain injections. Subjecting zebrafish larvae to

11 a novel TBI paradigm produced various TBI symptoms including cell death,

12 hemorrhage, blood flow abnormalities, post-traumatic seizures, and Tau inclusions.

13 Bath application of anticonvulsant drugs rescued TBI-induced tauopathy and cell death;

14 these benefits were attributable to inhibition of post-traumatic seizures because co-

15 application of convulsants reversed these beneficial effects. However, one convulsant

16 drug, 4-Aminopyridine, unexpectedly abrogated TBI-induced tauopathy - this was due to

17 its inhibitory action on endocytosis as confirmed via additional dynamin inhibitors. These

18 data suggest a role for seizure activity and dynamin-dependent endocytosis in the prion-

19 like seeding and spreading of tauopathy following TBI. Further work is warranted

20 regarding anti-convulsants that dampen post-traumatic seizures as a route to

21 moderating subsequent tauopathy. Moreover, the data highlight the utility of deploying

22 in vivo Tau biosensor and TBI methods in larval zebrafish, especially regarding drug

23 screening and intervention. 


\section{Introduction:}

26 Traumatic brain injury (TBI) is a leading cause of mortality and disability worldwide

27 (Hay et al., 2016; Nguyen et al., 2016; Rimel et al., 1981). It also is a prominent risk

28 factor for neurodegeneration and dementia, such as chronic traumatic encephalopathy

29 (CTE) (Chauhan, 2014; Gardner and Yaffe, 2015; Uryu et al., 2007). TBI can result from

30 direct physical insults, from rapid acceleration and deceleration of the brain, or from

31 shock wave impacts such as explosive blasts (Cruz-Haces et al., 2017). Regardless,

32 the primary mechanisms have much in common and the neuropathology in TBI and

33 CTE patients includes the wide distribution of hyperphosphorylated tau pathology,

34 axonal degeneration, and neuronal loss (Hay et al., 2016; Johnson et al., 2013; McKee

35 et al., 2015; Ojo et al., 2016). The mechanisms whereby physical injury is translated into

36 progressive tau pathology remain unresolved and represent prospective therapeutic

37 targets. Progress on this front is hampered by lack of access to suitable models:

38 applying physical injury to a cell culture is difficult and poorly represents the complex

39 biopathology that intertwines many multifaceted aspects of brain physiology.

40 The progressive deposition of hyperphosphorylated tau protein in filamentous forms

41 is a defining hallmark of tauopathies, which includes Alzheimer's disease (AD), CTE,

42 and several other dementias. Each of the tauopathies affects distinct brain regions and

43 has a unique clinical presentation (Kovacs, 2017; Orr et al., 2017). Early in CTE,

44 hyperphosphorylated tau is accumulated in a cluster of perivascular neurons and glia in

45 the depths of cortical sulci. Later in CTE, tau pathology is widespread and incorporates

46 cortical and subcortical grey-matter areas (Hay et al., 2016; Johnson et al., 2012;

47 McKee et al., 2015). This broad spreading of tau pathology in CTE can also be 
observed following TBI ascribed to single trauma events (Johnson et al., 2012). This spreading of tauopathy is consistent with a prion-like mechanism; indeed brain homogenates from mice subjected to TBI can initiate p-Tau pathology when injected

51 into healthy wildtype mice (Zanier et al., 2018). The recipient mice develop a p-tau

52 pathology similar to single severe TBI patients, which then spreads from injection sites

53 to distant regions, behaving similarly to bona fide prions (Zanier et al., 2018).

54 Beyond TBI, the self-propagation and prion-like spread of tau aggregates is thought

55 to play a key role in the progression of other tauopathies such as AD (Iba et al., 2013;

56 Iba et al., 2015; Mudher et al., 2017; Narasimhan et al., 2017; Sanders et al., 2014).

57 Mechanisms of tau spreading, and the therapeutic targets they offer, have principally

58 been defined in vitro and include tunnelling nanotubes and extracellular vesicles (EVs

59 such as exosomes and synaptic vesicles) and their uptake via endocytosis (Colin et al.,

60 2020; Demaegd et al., 2018; Evans et al., 2018). In AD and other tauopathies,

61 observations from patients and mice have highlighted the capacity of tau seeds to

62 spread trans-synaptically (Goedert et al., 1989; Pickett et al., 2017). Moreover, it has

63 been shown that neuronal activity serves an important role in the spread of tau

64 pathology and general proteostasis (Pickett et al., 2017; Wu et al., 2016; Yamada et al., 65 2014). Stimulation of neuronal activity increased the extracellular release of tau to the 66 media in vitro, and enhanced tau pathology in a mouse model of familial frontotemporal

67 dementia (Pickett et al., 2017; Wu et al., 2016; Yamada et al., 2014). Whether similar 68 mechanisms of tau release and spread occur following TBI remains unknown.

69 In this light, an intriguing aspect of TBI is the prominence of post-traumatic seizures

70 that might be predicted to initiate the aggregation and/or exacerbate the spread of tau 
71 pathology. Seizures are one of the key consequences of all types of TBI, and they have

72 been more commonly reported in patients who suffered from blast injuries (Asikainen et

73 al., 1999; Salinsky et al., 2015). Though the exact prevalence remains undetermined

74 (Lucke-Wold et al., 2015), it is anticipated that over 50\% of TBI patients with severe

75 injuries develop seizures or post-traumatic epilepsy (Kovacs et al., 2014). A link

76 between seizures and tau pathology is suggested by increased prevalence of seizures

77 in AD patients and animal models of AD (Sanchez et al., 2018; Yan et al., 2012).

78 Whether reducing post-traumatic seizures can delay or minimize the progression of

79 tauopathy has yet to be fully explored.

80 This knowledge gap is due in part to a lack of accessible in vivo models that can

81 report the progression and spread of tauopathy, or that allow neural activity associated

82 with TBI to be measured and manipulated. To address these issues, we engineered a

83 tauopathy biosensor transgenic zebrafish that develops GFP+ puncta when tau

84 aggregates within the brain or spinal cord. Additionally, we introduce a simple medium-

85 throughput method to induce TBI in zebrafish larvae. Combining these novel

86 approaches, we found that post-traumatic seizures correlate strongly with spreading tau

87 pathology following TBI. Manipulating this seizure activity mitigated tau aggregation and

88 revealed a critical role for endocytosis in the prion-like spread of tau seeds in vivo

89 following TBI. The results from our novel in vivo TBI model implicate seizures and

90 dynamin-dependent endocytosis in the spread of tau seeds, thereby offering potential

91 therapeutic targets.

92 


\section{Methods:}

\section{Animal Ethics and Zebrafish Husbandry}

96 Zebrafish were raised and maintained following protocol AUP00000077 approved by the

97 Animal Care and Use Committee: Biosciences at the University of Alberta, operating

98 under the guidelines of the Canadian Council of Animal Care. The fish were raised and

99 maintained within the University of Alberta fish facility under a 14/10 light/dark cycle at

$10028^{\circ} \mathrm{C}$ as previously described (Westerfield, 2000).

102 Generating Transgenic Tauopathy Reporter Zebrafish

103 To engineer the transgenic Tau4R-GFP reporter zebrafish, the human wild-type MAPT

104 sequence of the four-microtubule binding repeat domain (aa 244-372 of the full-length

105 TAU 2N4R, NCBI NC_000017.11, protein id NP_005901) with a seven-amino acid C-

106 terminal linker (RSIAGPA) was ordered as a gene block from IDT. The gene block was

107 subcloned into a middle entry cloning vector (Multisite Gateway® technology,

108 ThermoFisher). This was recombined with the p5E-enolase2 and p3E-GFP components

109 into destination vector pDestTol2CG2 of the Tol2kit (Guo and Lee, 2011; Kwan et al.,

110 2007). The destination vector contained a reporter construct [encompassing EGFP

111 driven by the cardiac myosin light chain (clmc) promoter that helps identify stable

112 transgenic zebrafish]. The resulting plasmid pDestTol2CG2.eno2:Tau4R-GFP was

113 delivered in a $10 \mu \mathrm{l}$ injection solution including $750 \mathrm{ng} / \mu \mathrm{l}$ of the construct mixed with

$114250 \mathrm{ng} / \mu \mathrm{l} \mathrm{Tol} 2$ transposase mRNA, $1 \mu \mathrm{l}$ of $0.1 \mathrm{M} \mathrm{KCL}$, and $20 \%$ phenol red. The solution

115 was injected into the single-cell embryos of Casper zebrafish line (transparent zebrafish

116 line)(White et al., 2008). Injected embryos were screened for mosaic expression of the 
117 Tau4R-GFP transgene at two days post-fertilization (dpf) using a Leica M165 FC

118 dissecting microscope. F0 mosaic fish were raised to adulthood and outcrossed.

119 Successful F1 embryos were identified by their abundant expression of Tau4R-GFP in

120 the CNS and the green heart marker. The stable transgenic line

121 Tg(eno2:Hsa.MAPT_Q244-E372-EGFP) ${ }^{\text {uа3171 }}$ was assigned the allele number ua3171.

122 An equivalent transgenic zebrafish biosensor was engineered to detect human

123 SOD1 aggregation. Subcloning from existing vectors (Pokrishevsky et al., 2018)

124 produced pDestTol2CG2.eno2:SOD1-GFP and similar transgenesis methods

125 engineered the Tg[eno2:SOD1-GFP] zebrafish line that was assigned allele number

126 ua3181.

127

128 Cell culture and Generation of Tauopathy Reporter Stable Cell Line

129 To move the Tau4R-GFP reporter above into a vector appropriate for cell culture,

130 BamHI and Xhol nuclease restriction enzymes were employed to remove the Tau4R-

131 GFP fragment from pDest tol2CG2.eno2.Tau4R-GFP.pA. The Tau4R-GFP fragment

132 was subcloned into the pCDNA3.1 vector using a T4 DNA ligase enzyme. Sequencing

133 of the cloned vector with the following reverse primer for GFP

134 (TCTCGTTGGGGTCTTTGCTC) confirmed the proper orientation. Purification of the

135 plasmid was conducted with the Qiagen purification kit. HEK293T cells were grown in

136 Dulbecco's modified Eagle's medium (GibcoTM, ThermoFisher) supplemented with

$13710 \%$ fetal bovine serum and $1 \%$ penicillin/streptomycin. All cells were maintained at

$13837^{\circ} \mathrm{C}$ in a humidified $5 \% \mathrm{CO} 2$ incubator. For passaging cells, cells were washed with 
139 phosphate-buffered saline (PBS) before trypsinization with 0.05 Trypsin-EDTA (Sigma 140 Aldrich, T4174).

141 HEK293T cells were plated at $1 \times 10^{6}$ cells/well in six-well plates. Cells were

142 transfected with pcDNA3.1.Tau4R-GFP plasmid 24 hours after plating using

143 lipofectamine 2000 reagents according to the manufacturer's guidelines. Briefly, $4 \mu \mathrm{g}$ of

144 pcDNA3.1.Tau4R-GFP was diluted in $250 \mu \mathrm{l}$ of Opti-MEM media (GibcoTM,

145 ThermoFisher). The expression of the fluorescent reporter was confirmed the next day

146 through microscopic analysis. A stable cell line was established by replating the

147 transfected cells at a 1:10 dilution and selecting in DMEM media containing $1200 \mu \mathrm{g} / \mathrm{ml}$

148 geneticin (GibcoTM, ThermoFisher). Expression of the fused fluorescent proteins in the

149 stable cell lines was confirmed using fluorescent microscopy. Polyclonal cells and

150 monoclonal cells were grown to confluency in $10 \mathrm{~cm}$ dishes, then stored in liquid

151 nitrogen until use.

153 Immunoblotting of Cell Lysate and Zebrafish Brain Lysate

154 For cell lysate preparation, cells were washed with cold PBS, then collected and 155 incubated with cold lysis buffer (150mM NaCl, 50mM Tris-HCl (pH 8), 1 mM EDTA and $1561 \%$ Nonidet P-40) supplemented with protease inhibitor (Cocktail Set III; Millipore) for 10

157 mins on ice. Cells were lysed using a bio-vortexer homogenizer for 20 sec for two 158 rounds. The lysate was centrifuged at $13000 \mathrm{rpm}$ for $10 \mathrm{mins}$ at $4^{\circ} \mathrm{C}$. The supernatant 159 was collected, and the protein concentration was determined using the Qubit ${ }^{\mathrm{TM}}$ Protein 160 Assay Kit (Invitrogen). 
For zebrafish brain lysate preparation, the brains of adult zebrafish were dissected.

162 Brains were homogenized in cell lysis buffer (20mM HEPES, 0.2mM EDTA, 10mM

$163 \mathrm{NaCl}, 1.5 \mathrm{mM} \mathrm{MgCl} 2,20 \%$ glycerol, $0.1 \%$ Triton-X) with protease inhibitor and

164 phospSTOP (Sigma-Aldrich) in the case of pt406 Tg. Brains were lysed using a bio-

165 vortexer homogenizer and sonicated for $3 \mathrm{sec}$ for one round. Samples were centrifuged

166 as above and concentration of the samples was assessed in a Qubit® fluorometer

167 (Invitrogen).

168 For immunoblotting, $30-40 \mu \mathrm{g}$ of the total protein was combined with $2 \mathrm{X}$ sample 169 buffer (Sigma-Aldrich) and boiled for 10 mins before loading in 11\% SDS-PAGE.

170 Electrophoresis was performed using the Bio-Rad Power PAC system in running buffer 171 (25 mM Tris base, $192 \mathrm{mM}$ glycine and 0.1\% SDS). The gel was transferred to a PVDF 172 membrane using a wet transfer system. All membranes were blocked for one hour in 173 protein-free blocking buffer PBS (ThermoFisher) or TBST with $5 \%$ milk and then 174 incubated with primary antibody overnight at $4^{\circ} \mathrm{C}$ with gentle agitation. The primary 175 antibodies used in this study include rabbit monoclonal GFP (abcam, EPR14104) at 176 1:3000 dilution, rabbit anti- $\beta$-actin (Sigma-Aldrich, A2066) at 1:10000. All membranes

177 were washed three times with $1 \mathrm{X}$ TBST before incubation with secondary antibody 178 (goat-anti-mouse HRP or HRP-conjugated anti-rabbit at 1:5000 dilution (Jackson 179 ImmunoResearch) for one hour at room temperature. The membranes were washed for 180 the final time before visualization using Pierce ${ }^{\circledR}$ ECL Western Blotting Substrate 181 (ThermoFisher) on a ChemiDoc (Biorad). For stripping and re-probing, the membranes 182 were stripped using mild stripping buffer (199.8 mM Glycine, 0.1\% SDS and 1\% Tween 18320 with a $\mathrm{pH}$ of 2.2) before blocking them and repeating the methods described before. 


\section{Immunohistochemistry}

185 Larvae were fixed overnight in $4 \%$ paraformaldehyde, either one day after being

186 subjected to TBI or following the subsequent application of drugs as indicated.

187 Immunostaining of Activated-Caspase3 on whole-mount larvae was carried out as

188 previously described (DuVal et al., 2014). Larvae were washed with 0.1 M PO4 with 5\%

189 sucrose three times before washing with $1 \%$ Tween in $\mathrm{H}_{2} \mathrm{O}(\mathrm{pH} 7.4)$, and then $-20^{\circ} \mathrm{C}$

190 acetone. Larvae were incubated in PBS3+ containing $10 \%$ normal goat serum for one

191 hour and then incubated with primary antibody with $2 \%$ normal goat serum in PBS3+.

192 The primary antibody used was polyclonal Anti-Active-Caspase-3 (BD Pharmingen,

$193559565)$ at 1:500 dilution. The secondary antibody applied was Alexafluor 647 anti-

194 rabbit at 1:200 dilution (Invitrogen). Larvae were counterstained with 4',6-diamidino-2-

195 phenylindole (DAPI) (ThermoFisher) for 30 minutes.

197 Preparations of Mouse Brain Homogenate (Crude and PTA Precipitated)

198 Brains from TgTau$^{\mathrm{P} 301 \mathrm{~L}}$ mice and non-Tg littermate controls (129/SvEvTac genetic

199 background) were provided by Dr. David Westaway and Dr. Nathalie Daude (Eskandari-

200 Sedighi et al., 2017; Murakami et al., 2006). Crude brain homogenate was prepared by

201 homogenizing the brains to $10 \%$ (wt/vol) in calcium- and magnesium-free DPBS that

202 included a protease inhibitor and phosSTOP, using a glass homogenizer and power gen

203 homogenizer (Fisher Scientific). Samples were then centrifuged at 13000 rpm for 15

204 mins at $4^{\circ} \mathrm{C}$. The clear supernatant was collected, aliquoted and stored in $-80^{\circ} \mathrm{C}$ until

205 use for experiments. 
The phosphotungstate anion (PTA)-precipitated brain homogenate was prepared as described (Woerman et al., 2016). Briefly, 10\% (wt/vol) brain homogenate was prepared as reported above and mixed with a final concentration of $2 \%$ sarkosyl (Sigma Aldrich)

209 and $0.5 \%$ benzonase (Sigma Aldrich, E1014), and then incubated at $37^{\circ} \mathrm{C}$ for two hours

210 with constant agitation in an orbital shaker. Sodium PTA (Sigma Aldrich) was dissolved

211 in $\mathrm{ddH}_{2} \mathrm{O}$, and the $\mathrm{pH}$ was adjusted to 7.0 before it was added to the samples at a final

212 concentration of $2 \%(\mathrm{vol} / \mathrm{vol})$. The samples were then incubated overnight under the

213 previous conditions. The next day, the samples were centrifuged at 16,000g for 30 mins

214 at room temperature. The supernatant was discarded, while the resulting pellet was

215 resuspended in $2 \%$ (vol/vol) PTA in $\mathrm{ddH}_{2} \mathrm{O}(\mathrm{pH} 7.0)$ and $2 \%$ sarkosyl in DPBS. The 216 samples were next incubated for one hour before the second centrifugation. The

217 supernatant was removed and the pellet was re-suspended in DPBS. An aliquot of $5 \mu l$ 218 of PTA purified brain homogenate was employed for electron microscopy (EM) analysis 219 to confirm the presence of fibrils in each sample.

221 Tau Fibrillization and EM Analysis:

222 Synthetic human tau protein (wildtype full-length monomers) was purchased as a 223 lyophilized powder (rPeptide, T-1001-2) and resuspended in $\mathrm{dd}_{2} \mathrm{O}$ at a concentration 224 of $2 \mathrm{mg} / \mathrm{ml}$. The recombinant protein was fibrillized as described previously (Guo and 225 Lee, 2011). Recombinant tau was incubated with 40uM low-molecular-weight heparin 226 and $2 \mathrm{mM}$ DTT in $100 \mathrm{mM}$ sodium acetate buffer $\left(\mathrm{pH} \mathrm{7.0)}\right.$ at $37^{\circ} \mathrm{C}$, thereafter being 227 agitated for seven days. The fibrillization mixture was centrifuged at $50,000 \mathrm{~g}$ for 30 
228 mins, and the resulted pellet was resuspended in $100 \mathrm{mM}$ sodium acetate buffer $(\mathrm{pH}$

229 7.0) without heparin or DTT. Successful fibrillization was verified by EM.

$230 \quad$ Negative staining for EM analysis of fibrils was conducted as described elsewhere

231 (Eskandari-Sedighi et al., 2017). Briefly, 400 mesh carbon-coated copper grids

232 (Electron Microscopy Sciences) were glow-discharged for $40 \mathrm{sec}$ before adding the

233 sample aliquots. PTA-purified brain homogenates or synthetic tau fibrils $(5 \mu L)$ were

234 applied on the top of the grid for $1 \mathrm{~min}$. These grids were washed using $50 \mu \mathrm{L}$ each of

$2350.1 \mathrm{M}$ and $0.01 \mathrm{M}$ ammonium acetate and negatively stained with $2 \times 50 \mu \mathrm{L}$ of filtered $2 \%$

236 uranyl acetate. After removing excess stain and drying, the grids were examined with a

237 Tecnai G20 transmission electron microscope (FEl Company) with an acceleration

238 voltage of $200 \mathrm{kV}$. Electron micrographs were recorded with an Eagle $4 \mathrm{k} \times 4 \mathrm{k} C \mathrm{CD}$

239 camera (FEI Company).

241 Liposome-Mediated Transduction of Brain Homogenate into Tauopathy Reporter

242 Cells

243 Polyclonal Tau4R-GFP cells were plated at $2 \times 10^{5}$ per well in 24 -well plates. Cells were

244 transduced the next day, using $40 \mu$ of $10 \%$ clarified brain homogenate combined with

245 Opti-MEM to a final volume of $50 \mu \mathrm{l}$. A further $48 \mu \mathrm{l}$ of Opti-MEM and $2 \mu \mathrm{l}$ of

246 Lipofectamine-2000 (Invitrogen) was added to the previous Opti-MEM mixture to a total

247 volume of $100 \mu \mathrm{l}$ and incubated for 20 mins. The liposome mixture was applied to the

248 cells for 18 hours, and cells were then washed with PBS, trypsinized, and re-plated on

249 coated coverslips (ThermoFisher) for imaging and analysis. 
For PTA-precipitated brain homogenate, 1:10 dilution of precipitated fibrils was used

251 for the transfection. $5 \mu$ l of PTA-purified fibrils was diluted in $45 \mu$ Opti-MEM to a final

252 volume of $50 \mu \mathrm{l}$. The previous Opti-MEM mixture was added to $47 \mu \mathrm{l}$ of Opti-MEM and

$2533 \mu \mathrm{l}$ of Lipofectamine-2000 and incubated in room temperature for 2 hours as described

254 in (Safar et al., 1998; Woerman et al., 2016). The mixture was added to cells, washed

255 after 18 hours and re-plated before analysis exactly as mentioned previously.

\section{Quantification of the Percentage of Cells with Positive Inclusion}

258 Prior to imaging, transfected cells were fixed 2\% PFA in PBS for 15 mins. Samples

259 were then washed twice with PBS then stained with DAPI (1:3000 from $1 \mathrm{mg} / \mathrm{ml}$ stock)

260 for six mins. Cells were imaged using a Zeiss LSM 700 scanning confocal microscope

261 featuring Zen 2010 software (Carl Zeiss, Oberkochen, Germany). Due to increased

262 brightness of the GFP+ puncta formed after introduction of brain homogenate, GFP 263 exposure was minimized for those cells only. To quantify the GFP+ puncta, a total of

264 nine images were collected and analyzed for each condition, each with 100 cells.

265 DAPI-positive nuclei were utilized to determine the number of cells per image. The 266 number of cells with inclusions (multiple nuclear inclusions or one cytoplasmic puncta) 267 were counted and the percentage was calculated.

269 Brain Ventricle Injections into Tauopathy Reporter Larvae

270 Injections into the larval zebrafish brain (intraventricular space) were performed as

271 described previously with few modifications (Gutzman and Sive, 2009). Embryos at 2 $272 \mathrm{dpf}$ (days post-fertilization) were removed from their chorions and anesthetized with $4 \%$ 
273 tricaine (MS-222, Sigma Aldrich). The embryos were placed in a 1\% agarose-coated

274 dish with small holes. Under a stereomicroscope, the immobilized embryos were

275 oriented so that the brain ventricles were accessible for injections. The injection was

276 carried out via pulled capillary tubes mounted in a micromanipulator. The injection

277 volume was calibrated to $5 \mathrm{~nL}$ by injection into mineral oil and measurement with an

278 ocular micrometer. Thereafter, the needle containing the injection solutions was placed

279 through the roof plate of the hindbrain and $5-10 \mathrm{~nL}$ of either $10 \%$ clarified brain

280 homogenate $\left(\operatorname{TgTau}^{\mathrm{P} 301 \mathrm{~L}}\right.$ mice or wildtype littermate control), or synthetic tau, were

281 mixed with $20 \%$ dextran Texas Red fluorescent dye (Invitrogen) and injected into the

282 ventricles. For all the brain injection experiments, an uninjected control group and

283 control group injected only with $20 \%$ red dextran fluorescent dye in PBS were included.

284 After the injections, embryos were screened using a Leica M165 FC dissecting

285 microscope and appropriately injected larvae were gathered for further analysis. The

286 injections were considered appropriate if they had sharp edges and non-diffuse dye in

287 the ventricle (Fig. S2A). Larvae receiving improper injections, in which the needle was

288 inserted too deep in the brain ventricles resulting in the dye being visible outside the

289 ventricle space and/or in the yolk, were excluded from analysis.

291 Microscopy Analysis of GFP Positive Puncta in Tau Reporter Larvae

292 For the microscopic analysis of GFP-positive inclusions, larvae that were either injected

293 or treated with traumatic injury, along with the control groups, were anesthetized via

294 tricaine at the indicated time point (two, three, four, or five days post-injection (dpi) or

295 post traumatic injury (dpti) depending on the experiment). Images for GFP-positive 
296 puncta on the brain area or lateral line above the spinal cord were taken using a Leica

297 M165 FC dissecting microscope and the number of GFP-positive puncta were manually

298 counted.

299

300 Traumatic Brain Injury (TBI) paradigm for Zebrafish Larvae

301 To induce TBI, 10-12 unanesthetized larvae (3 dpf) were loaded into a 10ml syringe 302 with $1 \mathrm{ml}$ of E3 media. The syringe was blocked using a stopper valve to ensure no

303 larvae or media left the syringe upon compression of the plunger. The syringe was held 304 vertical using a metal tube holder at the bottom end of a 48" tube apparatus. A defined 305 weight (between 30 and $300 \mathrm{~g}$ ) was dropped manually from the top of the tube. The tube 306 diameter was matched to (slightly greater than) the weight's diameter to enhance 307 repeatability. This was either done once or repeated three times, with either 65 or $300 \mathrm{~g}$ 308 weights. Once larvae were subjected to the traumatic brain injury, they were moved 309 back to a petri dish with fresh media and maintained for further analysis.

\section{Quantifying the pressure induced during TBI}

312 To characterize the dynamic changes in pressure that occurred within the syringe

313 during the TBI events, the stopper valve attached to the syringe (described immediately

314 above) was replaced with a piezoresistive pressure transducer (\#MLT844 AD

315 Instruments, Colorado Springs, CO). Events were monitored via a PowerLab 2/26 data

316 acquisition device and LabChart 7 software (AD Instruments). The pressure transducer

317 was zeroed to report gauge pressure (pressure changes relative to atmospheric 318 pressure) and was calibrated against a manometer (Fisherbrand Traceable from 
319 Thermoscientific, Ottawa ON). After each weight drop, the syringe apparatus was reset

320 to remove any air bubbles and the pressure transducer was zeroed. Time courses of

321 induced pressure were reported over a $350 \mathrm{msec}$ time frame with $50 \mathrm{msec}$ of base line

322 recording, while mean and maximum pressure values were calculated from the initial

323300 msec following the impact of the weight.

\section{Recording blood flow following TBI}

326 Abnormalities of blood flow and circulation resulted from TBI was detected 5 to 10 mins

327 after larvae was subjected to TBI. The blood flow in the tail area of zebrafish larvae,

328 either those subjected to TBI or uninjured controls, was recorded using Leica DM2500

329 LED optical microscope.

\section{Measuring the Seizure-like Phenotype in TBI Larvae}

332 The seizure-like behavior and activity of zebrafish larvae post traumatic injury

333 experiment was quantified via behavioral tracking software as described in our recent

334 publications (Kanyo et al., 2020; Leighton et al., 2018). Briefly, control larvae or larvae

335 subjected to traumatic brain injury using $65 \mathrm{~g}$ weight, were placed individually in wells of

336 96-well plates. The locomotor and seizure activity were assessed 40 minutes after the

337 traumatic brain injury through EthoVision ${ }^{\circledR}$ XT-11.5 software (Noldus, Wageningen,

338 Netherlands). The hypermotility of larvae is a manifestation of Stage I and Stage II 339 seizures (previously defined via application of epileptic drugs), whereas more intense

340 Stage III seizures are arrhythmic convulsions that manifest as reduced macroscopic 
341 movement in this assay (Kanyo et al., 2020; Leighton et al., 2018; Liu and Baraban,

342 2019).

\section{Engineering CaMPARI transgenic zebrafish for integrative calcium imaging}

345 The Tg[elavl3:CaMPARI (W391F+V398L)] ${ }^{\text {ua3144 }}$ zebrafish line expressing the calcium

346 sensor CaMPARI was generated using the Tol2 transgenesis system. We re-derived

347 these previously established CaMPARI transgenic fish due to a federal moratorium on

348 importing zebrafish into Canada (Hanwell et al., 2016). The Tol2 vector, pDestTol2-

349 elavl3:CaMPARI (W391F+V398L), was a gift from Eric Schreiter's lab and was

350 published in (Fosque et al., 2015). The Tol2 vector was injected in embryos at the 1-2

351 cell stage as previously described (Fisher et al., 2006). Transient larvae were identified

352 through green fluorescent cells in the central nervous system and were outcrossed to

353 obtain a stable Tg[elavl3:CaMPARI (W391F+V398L)] ua3144 F1 line. The F1 line was

354 crossed into the transparent Casper background.

356 Measuring neuronal activity during TBI using CaMPARI

357 Bright green CaMPARI larvae were loaded into $20 \mathrm{ml}$ syringe containing $1 \mathrm{ml}$ E3 media

358 (prepared as per Westerfield 2007, but without ethylene blue) and were exposed to a $359405 \mathrm{~nm}$ LED array (Loctite), which illuminated the syringe entirely (Fig. 4A). Larvae 360 were exposed for $10 \mathrm{sec}$, with the LED array at a distance of $7.5 \mathrm{~cm}$ from the syringe, 361 while subjected to traumatic brain injury using the $300 \mathrm{~g}$ weight as described above. 362 Following this photoconversion of CaMPARI during TBI, larvae were anesthetized in 
$3630.24 \mathrm{mg} / \mathrm{mL}$ tricaine (MS-222, Sigma Aldrich) and embedded in $2 \%$ low-gelling agarose

364 (A4018, Sigma Aldrich) for analysis under confocal microscopy.

365 Maximum intensity projections were acquired from Z-stacks (8 $\mu \mathrm{m}$ steps) using a

366 20x/0.8 Objective and a laser-point scanning confocal microscope (Zeiss 700). The

367 hindbrain area was analyzed, as it was the brain region most responsive to traumatic

368 brain injury. To specifically isolate the brain regions and obtain data points, a 3D area

369 was isolated by creating a surface with Imaris ${ }^{\circledR} 7.6$ (Bitman, Zuerich) and the mean

370 fluorescence intensities of the green and red channel intensities were calculated. Data

371 points were presented as a red/green ratio for each individual larva and interpreted as

372 relative neural activity, which is defined as red photoconverted CaMPARI in ratio to

373 green CaMPARI (Fosque et al., 2015) (R Kanyo, IN REVISION Jan 16th, 2020 ).

\section{Bath Application of Drugs}

376 Tau biosensor larvae were treated with $20 \mu \mathrm{M}$ of the proteasome inhibitor MG-132 at

377 2dpf, following injections with brain homogenate from Tg human Tau mice. The

378 treatment was left for 48 hours before changing the media and evaluating the

379 percentage of larvae developing GFP+ puncta in the brain region.

380 For Kainic acid or kainate treatment (KA), the appropriate doses $(5,50,100,150$,

381 and $200 \mu \mathrm{M}$ ) were added within 6 hours after TBI. For 4-aminopyridine (4-AP), one of

382 two doses of 4 -AP $(200$ or $800 \mu \mathrm{M})$ were added either six or 24 hours after TBI, as

383 indicated. For Retigabine (RTG) treatment, $10 \mu \mathrm{M}$ was used to treat TBI larvae

384 beginning six hours after TBI. Unless otherwise stated, KA, 4-AP and/or RTG were 
385 applied to larvae for 38 hours, then a fresh drug-free E3 media was added. The

386 formation of GFP-positive puncta was analyzed at four to five days post injury.

387 Pyrimidyn-7 ${ }^{\mathrm{TM}}(\mathrm{P} 7)$, the dynamin inhibitor, was purchased at a $50 \mathrm{mM}$ concentration

388 supplied in DMSO (Abcam). Larvae that were subjected to TBI were treated within six

389 hours following the injury with $3 \mu \mathrm{M}$ of $\mathrm{P} 7$. The dose was chosen based on the previous

390 use of the P7 drug on zebrafish larvae (Verweij et al., 2019). The larvae were incubated

391 with the drug for 20 hours, after which they were transferred to a fresh plate with drug-

392 free media. Dyngo 4a, another dynamin inhibitor (McCluskey et al., 2013), was

393 purchased from (Abcam) and $4 \mu \mathrm{M}$ of Dyngo 4a was used to treat larvae as previously

394 explained with P7. The formation and abundance of GFP-positive puncta was evaluated

395 as previously described at four days post traumatic injury (dpti). For some experiments,

396 the 'Tau biosensor' transgenic zebrafish were bred to a separate Tg line that express

397 human four repeat TAU Tg(eno2:hsa.MAPT-ires-egfp $)^{\text {Pt406 }}$ throughout the zebrafish

398 CNS (Bai et al., 2007).

\section{Statistics}

$401 \quad$ All statistical analyses were performed using GraphPad Prism Software (Version 7,

402 GraphPad, San Diego, CA). Sample sizes appropriate for our conclusions were

403 estimated iteratively as the variance in each of our new methods became apparent;

404 dose-response curves and significant differences amongst these dose were used to 405 judge that any detected impacts of subsequent interventions would be valid. All 406 experiments were independently replicated at least twice, individual larvae were the 407 sampling unit (reported on Figures), and no outliers or other data were excluded. The 
experimenters were blinded to the treatments prior to quantifying outcomes. Unpaired t-

409 tests were used to compare between two groups. For comparison between three or

410 more groups at various time points or the same time point, 2-way and Ordinary One-

411 Way ANOVA were used followed by post-hoc Mann-Whitney $U$ tests and Kruskal-

412 Wallace multiple comparison tests, respectively.

\section{Results:}

\section{Engineering and Validation of Tauopathy reporter lines.}

Previous reports describe the assessment and quantification of tau inclusions in

419 living cells (typically Human Embryonic Kidney cells), via measuring aggregation of

420 fluorescent proteins fused to tau protein, providing sensitive detection of pathological 421 tau species and strain variants (Kaufman et al., 2016; Sanders et al., 2014; Woerman et

422 al., 2016). Tau is predominantly expressed in the neurons of the CNS and we reasoned

423 that fluorescent biosensor tools would have good potential to reveal additional

424 phenotypes when expressed in these cells and moreover, that prion-like mechanisms of

425 tauopathy spread are best modeled in an intact brain (e.g. vectored by blood and 426 glymphatic circulation, ventricles, axonal projections and immune systems). Therefore,

427 we engineered a tauopathy biosensor transgenic zebrafish that expresses a fluorescent

428 tau reporter protein. Our genetically encoded fluorescent reporter protein was

429 composed of the sequence of the human tau core-repeat domain fused to GFP with a 430 linker sequence and is referred to here as Tau4R-GFP (Fig. 1A and S1A). Contrasting

431 previous in vitro models, our biosensor did not feature any pro-aggregation mutations in 432 the human tau repeats; this design was intended to minimize spontaneous aggregation 
Fig 1. Validating tauopathy fluorescent biosensor in vitro and in zebrafish. The biosensor Tau4R-GFP was validated for its ability to detect tauopathy seeds in vitro and in zebrafish. (A) Schematic of Tau4R-GFP "Tau biosensor" that contains the four binding repeats $(4 R)$ region of wildtype human tau linked to green fluorescent protein (GFP; see also Fig S1A). (B) Transgenic zebrafish engineered to express Tau4R-GFP biosensor throughout neurons of the CNS. Wildtype GFP is also abundant in the heart, which serves as a marker of the transgene being present but is otherwise irrelevant to our analyses. (C) Western Blot on zebrafish brain confirmed production of Tau4R-GFP at the expected size, similar to a SOD1-GFP biosensor and coordinately larger than GFP alone. (D) Human Tau fibril precipitated from transgenic ( $\mathrm{Tg}$ $\mathrm{Tau}^{\mathrm{P} 301 \mathrm{~L}}$ ) mouse brain homogenates using PTA and assessed by EM. (E) Application of PTA-purified brain homogenate induced the formation of tau inclusions similar to clarified brain homogenate (scale bar 50 $\mu \mathrm{m}$; compare to Fig S1D), but application of equivalent preparations from non-Tg mice produced no GFP+ inclusions. (F-I) Tau biosensor zebrafish detects diseaseassociated human tau fibrils following intraventricular injection of brain homogenate. Crude brain homogenates were microinjected into the hindbrain ventricle of Tau4R-GFP zebrafish larvae at two days post-fertilization, and tau inclusions were analyzed at several time points. (F) Tau biosensor zebrafish larvae developed readily apparent GFP+ inclusions in the brain and spinal cord (Fig. S2) when injected with brain homogenate burdened with tau pathology (from Tg mice) but not from healthy brain homogenate ( $F^{\prime}$, from non-Tg mice). $F$ " inset shows many adjacent cells exhibiting GFP+ Tau aggregates. (G) Tau biosensor zebrafish injected with human tau fibrils (within Tg mouse brain homogenate) developed significantly more aggregates on the spinal cord compared to uninjected control and other control groups, including compared to wildtype mouse brain homogenate $(* * p \leq 0.01, * * * p \leq$ 0.001) (H) Same data as in G, expressed as the percentage of larval fish showing Tau aggregates in the spinal cord, 4 .
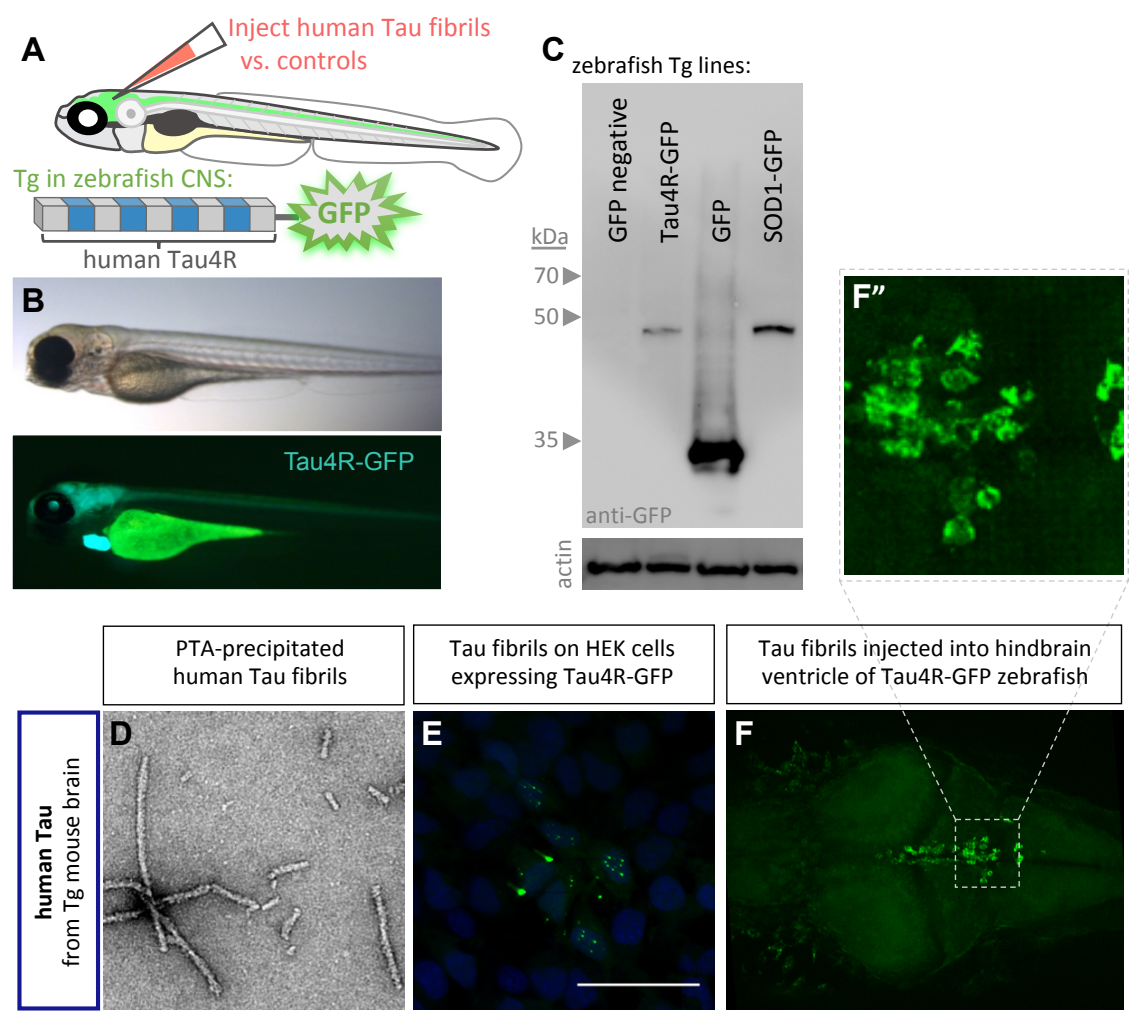

Tau fibrils injected into hindbrain ventricle of Tau4R-GFP zebrafish

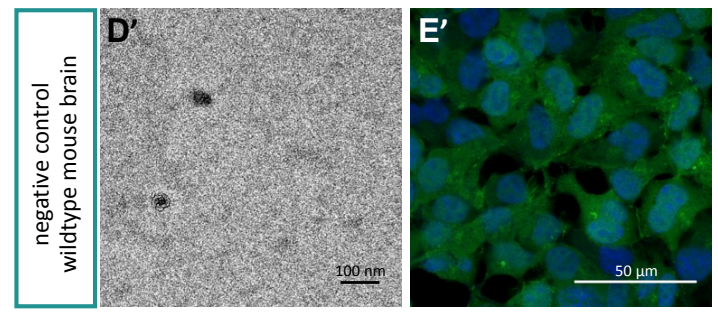

G Tau4R-GFP zebrafish injected w/ mouse brain homogenates:
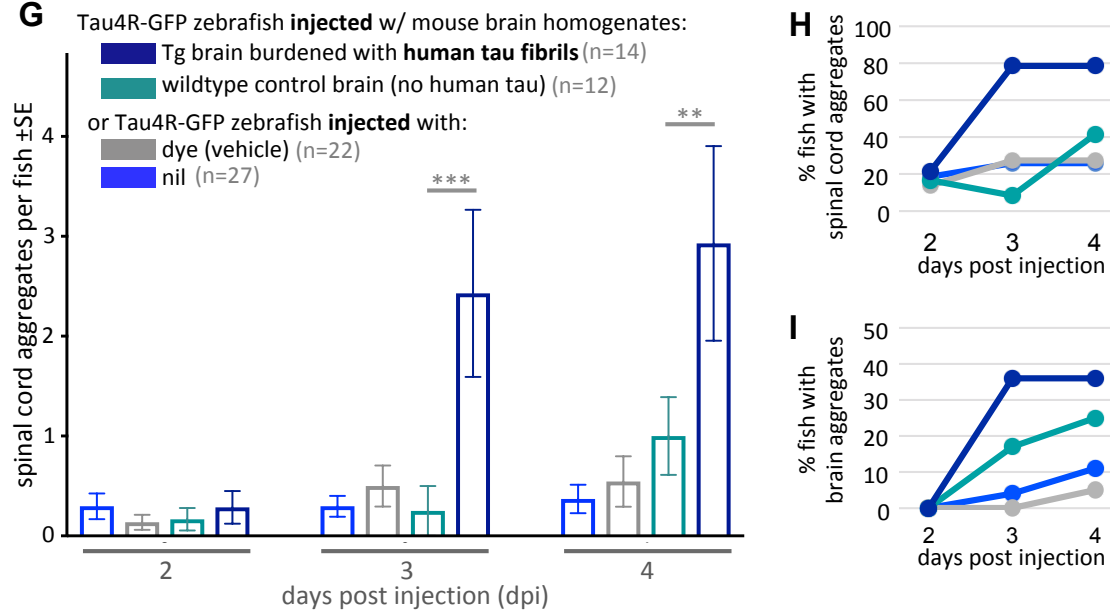
and (I) those same fish also showed Tau aggregates in the brain, over time. $n=$ number of individual larvae. 
434 events. The expression of the biosensor protein in zebrafish was under the control of

435 the pan-neuronal promoter neuronal enolase 2 (eno2, see Bai et al., 2007), which drives

436 expression throughout the CNS (Fig. 1B and S1C). We deployed the transgene in a

437 transparent zebrafish line (the 'Casper' background (White et al., 2008)) to facilitate

438 analysis beyond the early larval development stages (when pigmentation would

439 otherwise begin to obscure microscopy). We isolated a stable transgenic (Tg) line that

440 expresses the Tau4R-GFP biosensor reporter robustly and clearly in the CNS (Fig. 1B),

441 Tg(eno2:Hsa.MAPT_Q244-E372-EGFP) ${ }^{\text {ua3171 }}$, and assigned it allele number ua3171.

442 Simultaneously, we expressed the same biosensor in vitro to validate the construct

443 we deployed in vivo (Fig. S1A and B). Both in HEK293T cells and Tg zebrafish,

444 immunoblotting using anti-GFP antibody detected our Tau-4R-GFP reporter protein at

445 the expected size of $\sim 45 \mathrm{Kd}$, similar to a SOD1:GFP biosensor protein of similar

446 predicted size, and an appropriately larger size relative to GFP protein alone (Fig. 1C,

447 S1B').

448 We assessed the capacity of our Tau4R-GFP biosensor to report the presence of

449 tau pathology via transducing brain homogenates into cells. Brain homogenates

450 burdened with tauopathy, from transgenic mice expressing mutant human tau (Tg

$\left.451 \mathrm{Tau}^{\mathrm{P} 301 \mathrm{~L}}\right)$, were compared to normal non-Tg mouse homogenates as a negative control.

452 Congruent with findings obtained in past similar cell assays (Sanders et al., 2014), GFP-

453 positive (GFP+) inclusions were detected only when cells were transduced with brain

454 homogenate containing pathogenic human tau fibrils (from $\mathrm{Tg} \mathrm{Tau}^{\mathrm{P} 310 \mathrm{~L}}$ mice) (Fig.

455 S1D). The in vitro assay detection rate was approximately $5 \%$ of cells having GFP+

456 inclusions in total, with $2 \%$ of cells forming multiple nuclear puncta and $\sim 3 \%$ forming 
457 one cytoplasmic inclusion, whereas various negative controls consistently displayed $0 \%$

458 of cells with inclusions (Fig. S1E). To verify that tau aggregates in the clarified brain

459 homogenate caused the GFP+ puncta, we purified tau aggregates from the tissue 460 samples using PTA precipitations (Woerman et al., 2016). Tau fibrils purified from these

461 preparations were characterized via EM analysis (Fig. 1D). Transducing these

462 preparations (in contrast to control preparations derived from non-Tg mice) produced 463 fluorescent puncta in the Tau4R-GFP reporter cells (Fig. 1E), confirming the ability of 464 our Tau4R-GFP chimeric protein to report tau aggregation.

Validation of in vivo Tau biosensor via intra-ventricular brain injections of Tau

467 fibrils

To test if the Tau4R-GFP biosensor can report the in vivo progression of tauopathy,

469 we emulated intracerebral injection methods that induce (prion-like) tau pathology in 470 mice (Clavaguera et al., 2013; Guo et al., 2016; Peeraer et al., 2015). We injected

471 clarified brain homogenate laden with human tau fibrils, prepared as above from Tg 472 mice, into the hindbrain ventricle of two days post-fertilization (dpf) tau biosensor 473 zebrafish (Fig. S2A). The injected larvae and control groups were monitored daily for up 474 to four days post-injection (dpi). Biosensor larvae injected with human Tau fibrils (from 475 Tg mouse brain) developed GFP+ puncta, reflective of tau aggregation in the brain (Fig. 476 1F, F"). These tau inclusions were prominent near the ventricle wall as well as in 477 sensory neurons along the spinal cord, when injected with brain homogenate from 478 human-tau transgenic mouse (Fig. S2B). These puncta appeared to have either a lone 479 dot-like shape or were similar to the multiple nuclear puncta detected in vitro, in which 
480 three to four small puncta are clustered together. Repeated assessment of the location

481 of tau aggregates on the spinal cord of the same individuals over multiple days, using

482 somite numbers as landmarks, suggested a movement of some of these puncta over

483 time (Fig. S3A and B).

484 The abundance of GFP+ spinal cord inclusions was progressive and significantly 485 higher in larvae injected with pathogenic TAU brain homogenate compared with various 486 controls $(p<0.0001$ at $3 d p i$ and 4dpi, Fig. 1G). Few larvae in the control groups 487 developed spontaneous inclusions but the number of the larvae and the abundance of 488 those inclusions were minimal (Fig. 1G). $80 \%$ and $35 \%$ of the larvae injected with 489 human tau fibrils developed puncta in the brain and spinal cord, respectively (Fig. $2 \mathrm{H}$ 490 and I). On the other hand, a lower proportion of tau biosensor larvae developed 491 'spontaneous' inclusions post-injection with the control brain homogenate from wild-type 492 mice (Fig. 2H and I). Tau aggregates were detected on the spinal cord region as early 493 as 2 dpi. Intriguingly, a small percentage of larvae developed sporadic GFP+ tau 494 aggregates regardless of treatment. Visualizing the data as distributions of larvae with 495 particular abundances of GFP+ inclusions (Fig. S2C) highlights a trend where most 496 larvae did not develop aggregates unless they were injected with brain homogenate 497 containing fibrillar, pathogenic human tau species. In those cases, the biosensor larvae 498 developed an abundant number of aggregates. Overall, these data confirm the ability of 499 our biosensor model to detect pathogenic tau species in vivo.

$500 \quad$ Like other protein misfolding diseases, tauopathies reflect a proteostatic imbalance 501 wherein the clearance of pathological tau species is insufficient relative to accumulation 502 (Chiti and Dobson, 2006; Lim and Yue, 2015). We reasoned that if the tau biosensor 

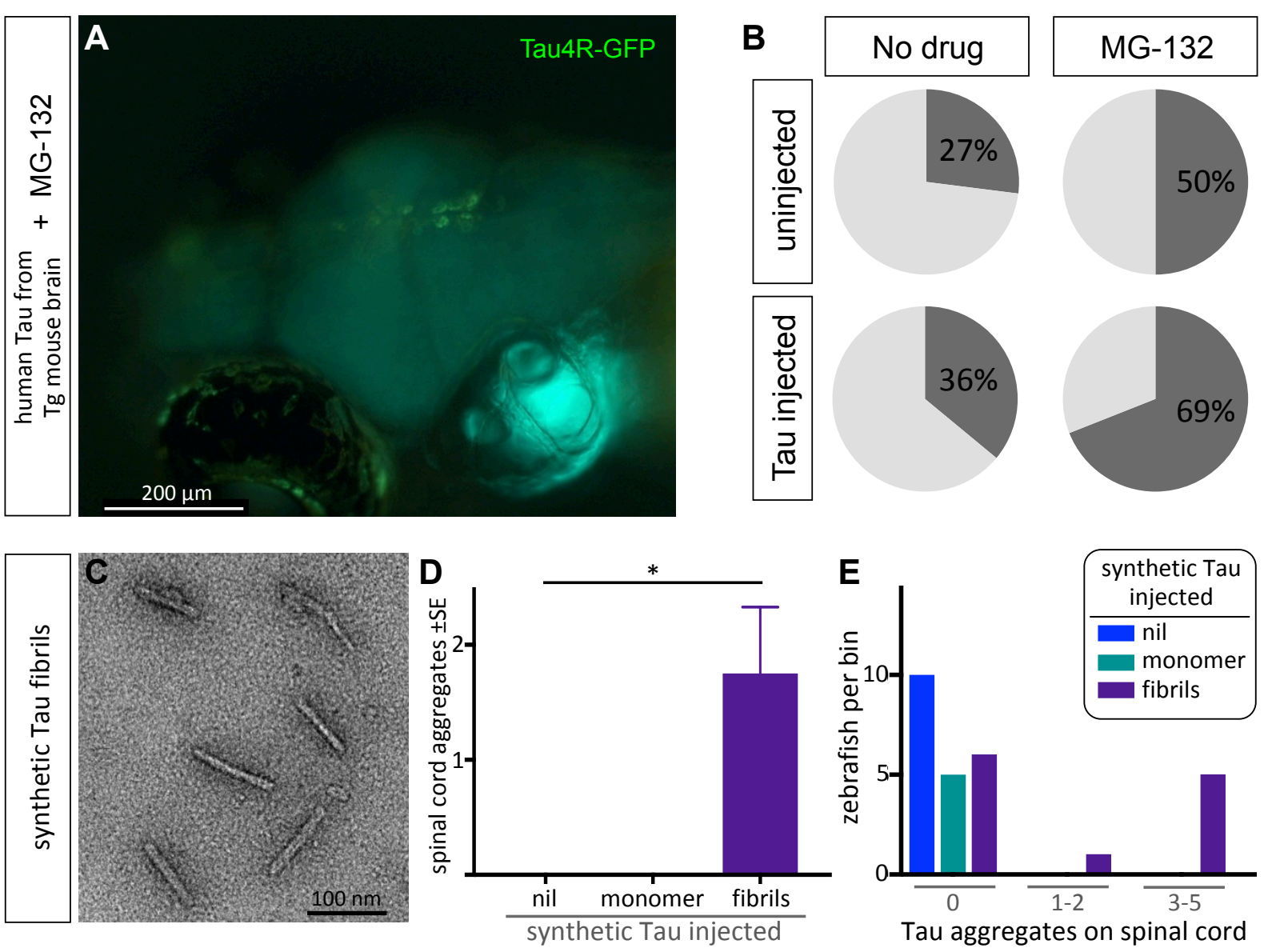

Fig 2. Protein-only induction of tau puncta in vivo detected in biosensor zebrafish. Injections of synthetic tau fibrils into Tau4R-GFP zebrafish induced GFP+ puncta in brains and spinal cord. $(\mathbf{A}, \mathbf{B})$ Inhibiting the proteosome with MG-132 enhanced the percentage of larvae bearing GFP+ inclusions in the brain following injection of tau-laden brain homogenate. (C) Synthetic human tau proteins were fibrillized as confirmed via EM analysis. Human Tau fibrils were microinjected into the larval hindbrain at two days post-fertilization, and tau inclusions were analyzed at three days post injections. (D) Tau aggregates were only observed after injection of tau fibrils, not monomers $\left({ }^{*} p<0.05\right)$. (E) Tau aggregates (same data as $D$, presented as distribution of larvae that displayed various amounts of GFP+ puncta) appear only after injection of tau fibrils, not monomers.

504 larvae are faithfully reflecting tau proteostasis concepts in vivo, then this could be

505 revealed via inhibition of the proteasome. We treated larvae with the proteasome

506 inhibitor MG-132 (Fig. 2A) and observed an approximate doubling of spontaneous 
507 GFP+ inclusions (Fig. 2B). Following injection of mouse brain homogenate containing 508 human tau fibrils, applying the proteasome inhibitor MG-132 substantially enhanced the

509 percentage of larvae bearing Tau4R-GFP+ inclusions in the brain (to 70\%, Fig. 2B),

510 relative to equivalent larvae without MG-132 ( 36\%, Fig. $2 B)$.

511 It was striking that the zebrafish tau biosensor was robustly able to discriminate

512 brain homogenates with human tau aggregates versus those that were not. However,

513 we considered an alternative explanation for the data: the difference may not depend

514 directly on human tau in the brain homogenate but could instead reflect other bioactive

515 components of the degenerating Tg mouse brain. To verify that the formation of GFP+

516 puncta in zebrafish can be seeded by a protein-only injection, we delivered synthetic

517 human tau protein (2N4R). After confirming the recombinant tau proteins were

518 appropriately fibrillized via EM (Fig. 2C), we delivered them by intraventricular injections

519 as described above. Similar to previous data with brain homogenate, the larvae that

520 were injected with synthetic tau fibrils developed inclusions proximal to the brain

521 ventricles as well as along the spinal cord at 3-6 dpi. The abundance of tau aggregates

522 along the spinal cord was significantly higher in larvae injected with the synthetic tau

523 fibrils compared to larvae injected with tau monomers or to the non-injected group

$524(p<0.05)($ Fig. 2D). The distribution of larvae based on the number of tau aggregates

525 they accumulated also supported these findings (Fig. 2E). In sum, the Tau4R biosensor

526 deployed in the CNS of larval zebrafish was able to report tau species, and further

527 revealed the prion-like induction of tauopathy via protein-only seeding in vivo. 


\section{Introduction of the first traumatic brain injury model for larval zebrafish}

531 We next sought to deploy our tau biosensor in a tauopathy model that enables

532 higher throughput than can be achieved with intraventricular injection methods. We

533 considered traumatic brain injury (TBI) as an inducer of the tauopathy in Chronic

534 Traumatic Encephalopathy (CTE); further, we were encouraged that innovations in this

535 realm could fill an unmet need for a high-throughput, genetically tractable in vivo model

536 of these devastating concussive injuries. Although a few methods have been reported to

537 induce traumatic brain injury in adult zebrafish that are comparable to mammalian TBI

538 methods (Maheras et al., 2018; McCutcheon et al., 2017), no such methods were

539 available for zebrafish larvae. Here we introduce and validate a simple and inexpensive

540 method to induce traumatic brain injury in zebrafish larvae. Investigating traumatic brain

541 injury in larvae offers substantial benefits regarding experimental throughput, economy,

542 accessibility of drug and genetic interventions, and bioethics.

543 We devised a traumatic injury paradigm by loading zebrafish larvae ( 12 individuals

544 in their typical E3 liquid growth media) into a syringe with a closed valve stopper, and

545 applying a hit on the plunger to produce a pressure wave through the fish body akin to

546 pressure or shock waves experienced during human blast injury (Nakagawa et al.,

547 2011) (Fig. 3A). To challenge the method's reproducibility, and to permit manipulation of

548 injury intensity, a series of defined masses were dropped on the syringe plunger.

549 Technical variability, anticipated from larvae being in different orientations and positions

550 within the syringe, was reduced by applying the injury three times to each group of

551 larvae (except where noted otherwise) while repositioning the syringe between each

552 injury. To assess if our method faithfully induced traumatic brain injury similar to injury 
Fig 3. Zebrafish larvae subjected to traumatic brain injury (TBI) exhibited various biomarkers of TBI. (A) A novel TBI model for larval zebrafish: To induce blast injury, zebrafish larvae were loaded into a syringe with a stopper. A defined weight was dropped on the syringe plunger from a defined height, producing a pressure wave through the fish body akin to pressure waves experienced during human blast injury. (B) Dynamics of the pressure increase after dropping weights of varying masses in our TBI model. (C,D) The mean and maximum pressures generated, respectively, by various weights applied in the TBI model. Dots represent individual trials. (E) Hemorrhage after TBI was observed in some of the larvae fish using Tg[gata1a:DsRed] transgenic zebrafish that express DsRed in erythrocytes, as indicated by white arrows. Lateral view of larval heads with anterior at the left. (F) Increased cell death in the brain of 4 dpf larvae subjected to TBI as indicated by immunostaining of activated Caspase-3 (magenta). Positive and negative controls for immunostaining are in Figure S4. Nuclei were stained with DAPI in
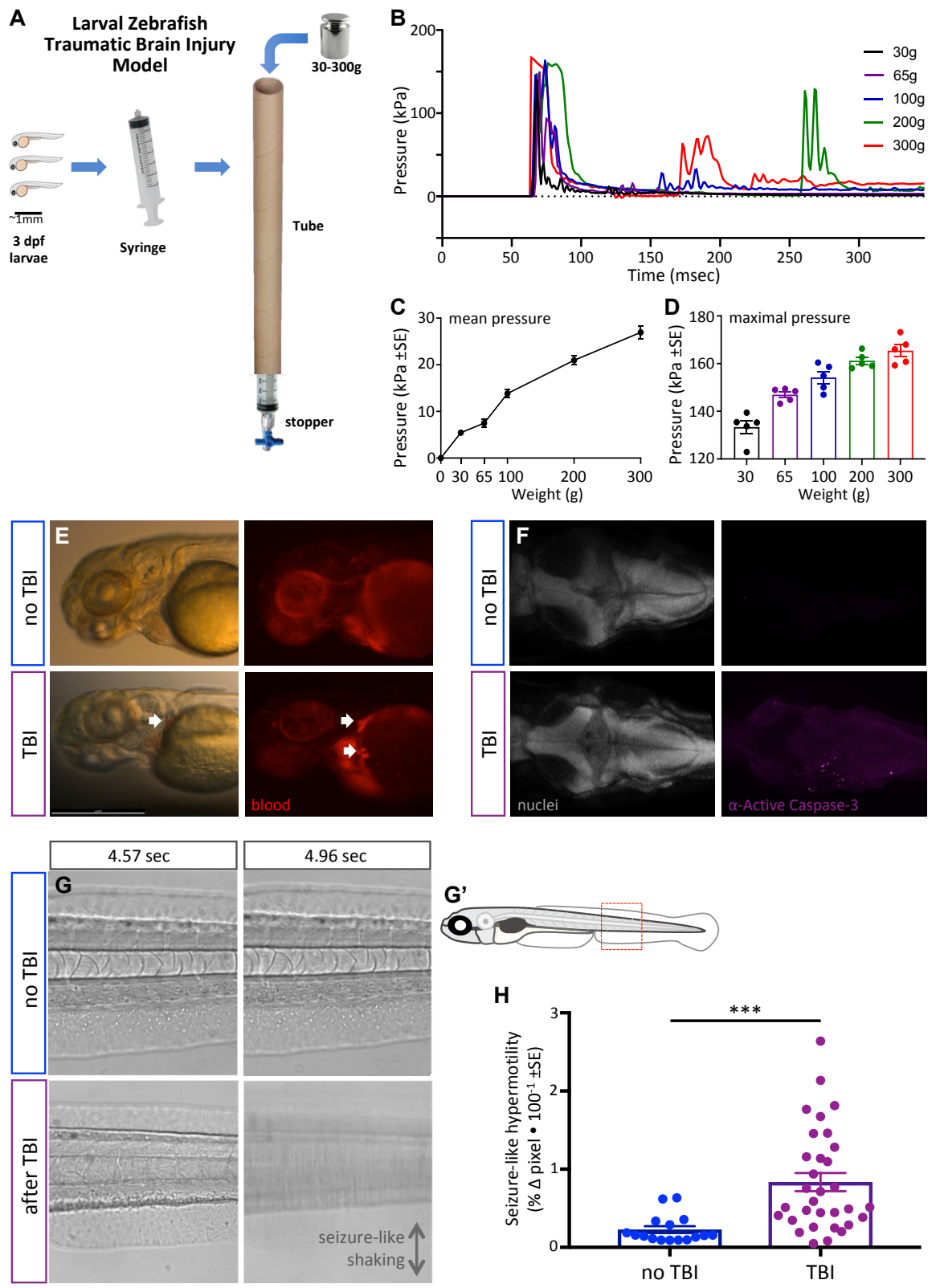
gray for reference. These are dorsal views of larval zebrafish brains with anterior at the left. (G) Seizurelike clonic shaking is observed in a subset of larvae after TBI. Movie frames are displayed from Supplemental Video S2. These frames (left and right panels) are separated by $\sim 400 \mathrm{msec}$ in time, and are lateral views of the larval zebrafish trunk (akin to red box in $\mathrm{G}^{\prime}$ ). Control fish without TBI show little movement except obvious blood flow. Following TBI, larvae show bouts of calm (bottom left) interspersed ( $\sim 00 \mathrm{msec}$ later) with bouts of intense seizure-like convulsions (Stage III seizures; bottom right). (H) Larvae subjected to TBI also displayed Stage II seizures, i.e. weaker seizures that manifest as hypermotility and are detected using a previously optimized behavioural tracking software system - seizures are significantly more intense following TBI compared to the control group $\left({ }^{* * *} p<0.001\right.$; dots are raw data for each larva, mean is plotted $\pm \mathrm{SE}$ ). 
555 from pressure waves, we examined multiple markers known to be associated with blast-

556 induced traumatic brain injury, including cell death, hemorrhage, blood flow

557 abnormalities, and tauopathy (Bir et al., 2012; Kovacs et al., 2014; Nakagawa et al.,

558 2011). Additionally, we evaluated the occurrence of post-traumatic seizure activity and

559 increases in neuronal activity acutely associated with the trauma.

560 We established the TBI method via empirical testing of various parameters,

561 restricting ourselves to materials and methods that can be adopted inexpensively, with a

562 goal of consistently inducing a robust injury (see phenotypes below) vs. a tradeoff with

563 maximizing survival of the larvae. Subsequent to this optimization, we were able to

564 characterize the pressure induced within the syringe during each injury (Fig. 3B-D). The

565 maximum pressure induced was near $170 \mathrm{kPa}$ (Fig. 3B). The dynamics of the pressure

566 change events during TBI (Fig. 3B) imply that dropping the heavier weights led to the

567 weight bouncing and producing a secondary increase in pressure (e.g. at $\sim 175$ or $\sim 275$

$568 \mathrm{msec}$ in Fig. 3B). The maximal pressure induced varied from $\sim 130$ to $\sim 175 \mathrm{kPa}$ in an

569 approximately linear fashion depending on the mass of the weight dropped (Fig. 3D).

570 The mean pressure change over the first $300 \mathrm{msec}$ of the TBI also increased in a nearly

571 linear fashion, and increased by nearly an order of magnitude when dropping weights of

$57230 \mathrm{~g}$ compared to $300 \mathrm{~g}$ (Fig. 3C).

573 We evaluated TBI-induced hemorrhage via the use of Tg[gata1a:DsRed] larvae that

574 have red fluorescence in their blood cells (Traver et al., 2003). Hemorrhage was

575 observed variably in larvae when a heavy weight $(300 \mathrm{~g})$ was used to induce the

576 traumatic injury (Fig. 3E). Further, approximately half of the TBI larvae showed

577 abnormalities in blood flow including a temporary reduction or complete absence of 
578 blood circulation (video 1), consistent with abnormalities detected in rodent TBI models

579 (Bir et al., 2012). Subsequently, we assessed apoptosis in the TBI larvae, observing

580 that our TBI method induced cell death in larvae as detected by staining for active

581 Caspase-3 (Fig. 3F and S4). The number of active-Caspase-3-positive cells was

582 negligible in the control groups compared to a mean of 62 apoptotic cells in TBI larvae

$583(\mathrm{SEM} \pm 9.17, \mathrm{n}=3)$ and $75(\mathrm{SEM} \pm 4, \mathrm{n}=2)$ in positive-control-larvae (cell death induced

584 with camptothecin, CPT; Fig. S4). These data all align well with existing animal models

585 of TBI with respect to mimicking characteristic features of human TBI, and support the

586 effectiveness of our method in inducing traumatic brain injury in larval zebrafish.

TBI treated larvae exhibited post-traumatic seizure-like behavior and increased

591 traumatic brain injuries and, despite being prevalent, remain poorly understood in TBI

592 patients (Kovacs et al., 2014). Post-traumatic seizures were overtly apparent in a subset

593 (approximately 40\%) of zebrafish larvae after they were subjected to traumatic brain

594 injury. In some instances the activity was highly reminiscent of Stage III seizures

595 (defined previously in larval zebrafish as the most intense seizures; Liu and Baraban,

596 2019) with bouts of intense clonic convulsions and arrhythmic shaking (Supplemental

597 Video S2; exemplar frames from the movie are in Figure 3G). Other individuals

598 exhibited hypermotility that is exactly consistent with past definitions of less intense

599 Stage I or Stage II seizures. We quantified the latter seizure activity via behavioral 600 tracking software (which we had previously optimized and validated for quantifying 
601 seizures in larval zebrafish (Kanyo et al., 2020; Leighton et al., 2018)) and determined 602 that larvae subjected to TBI exhibited seizure-like activity that was significantly higher 603 than the control group $(p<0.0007)($ Fig. $3 H)$.

604 Seizures are caused by abnormal and excessive neuronal excitability (Stafstrom 605 and Carmant, 2015). To document bursts of neuronal activity during the brain trauma, if 606 any, we utilized a genetically encoded calcium imaging CaMPARI reporter (calcium 607 modulated photoactivatable ratiometric integrator) expressed throughout the CNS. 608 CaMPARI fluoresces green in baseline conditions, and permanently converts to red 609 fluorescent emission if high intracellular calcium levels (i.e. neural activity) occur 610 coincident with application of 'photoconverting' intense 405nm blue light. We subjected 611 our allele of Tg[elav/3:CaMPARI] ${ }^{\text {ua3144 }}$ larvae (R Kanyo, IN REVISION Jan 16th, 2020 ) 612 to TBI, coincident with brief application of photoconverting light (405nm light provided by 613 an LED array directed at the syringe, as described in Fig. 4A). A sharp increase in 614 neuronal activity during TBI was evident, especially in the hindbrain region as indicated 615 by enhanced red emission (Fig. 4B). CaMPARI allows robust quantification of neural 616 activity expressed as a ratio of red:green fluorescent emission, which confirmed that 617 neuronal excitability increases significantly in response to brain trauma (Fig. 4C and D). 618 Notably, this combination of newly introduced methods of traumatic brain injury being 619 integrated with CaMPARI optogenetic methods (where the stable/irreversible changes 620 from green to red fluorescent reportage allows a ratiometric quantification in a 621 subsequent microscopy session) offers the rare ability to assess neural activity on un622 restrained (free-swimming) subjects during TBI. In sum, our data reveal a substantial 623 burst of neural activity occurs during TBI, and that zebrafish larvae exposed to TBI 

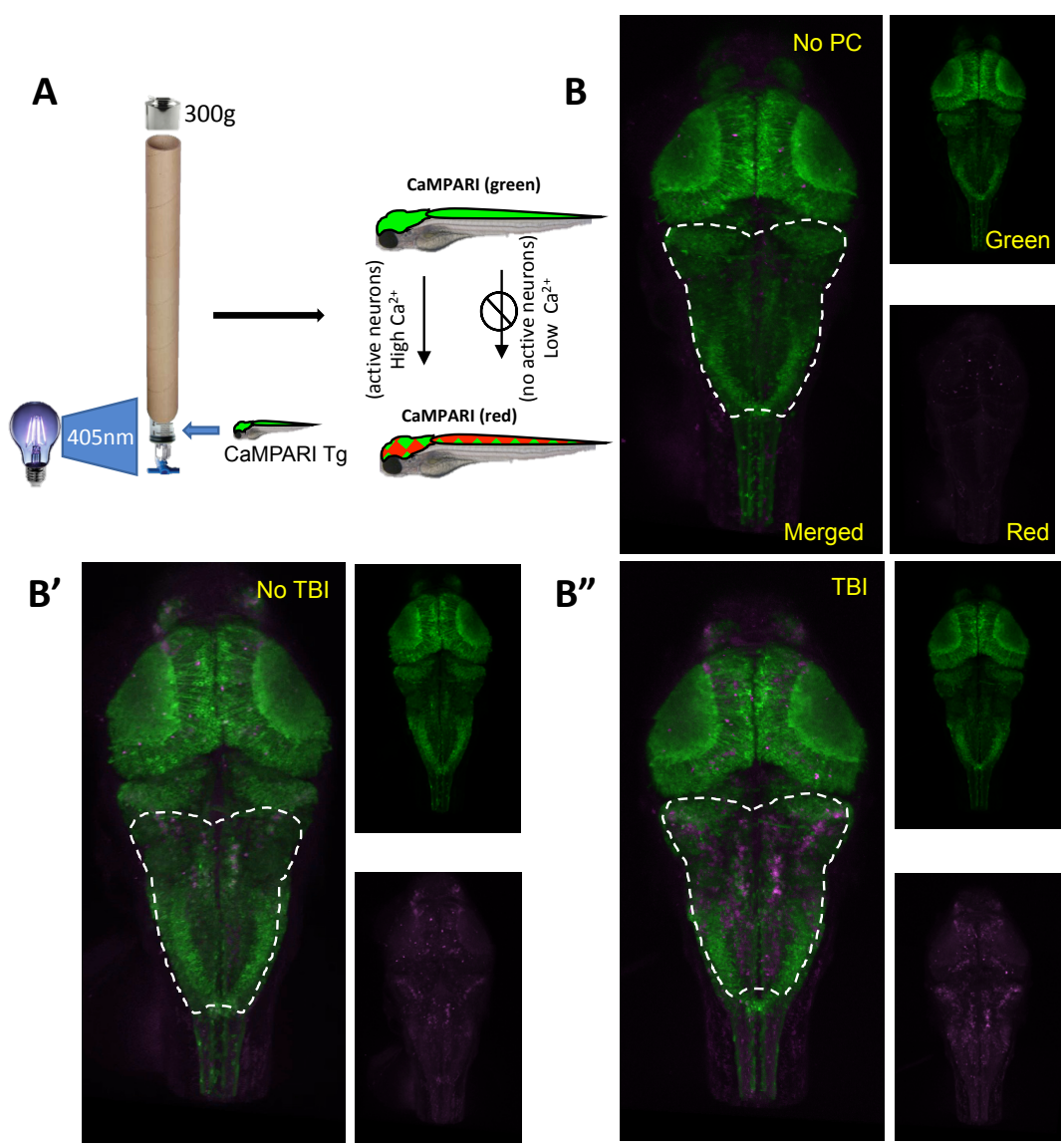

B"
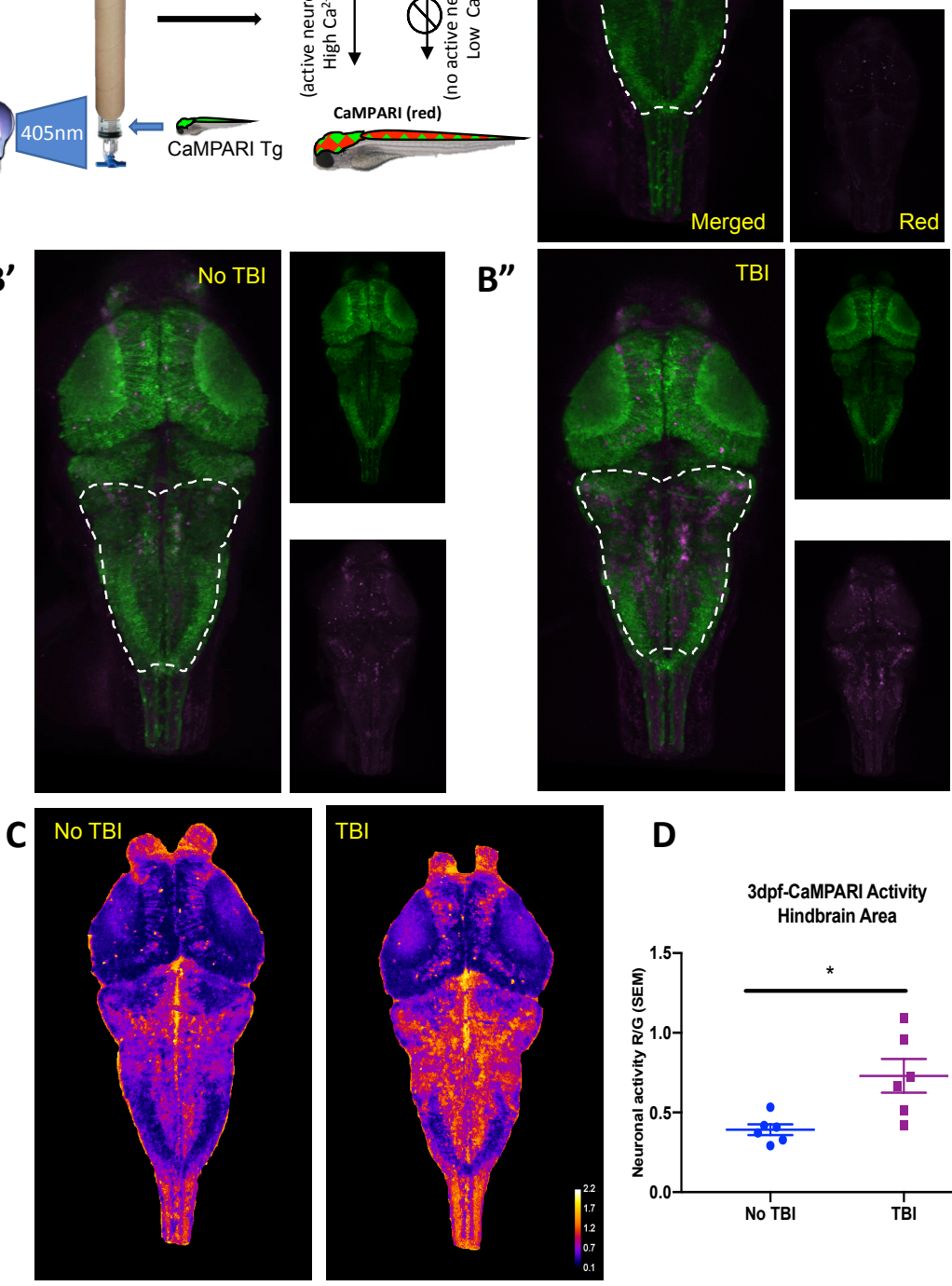

D

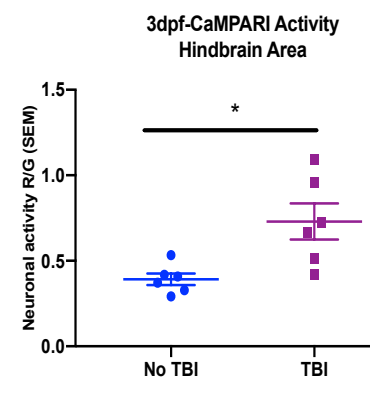

Fig 4. Neural activity increases during traumatic brain injury (TBI) as measured in CaMPARI zebrafish larva. (A) Schematic of TBI using CaMPARI (Calcium Modulated Photoactivatable Ratiometric Integrator) to optogenetically quantify neuronal excitability. 3dpf CaMPARI larvae were freely swimming while subjected to $\mathrm{TBI}$, coincident with exposure to $405 \mathrm{~nm}$ photoconversion light. CaMPARI fluorescence permanently photoconverts from green to red emission only if the photoconversion light is applied while neurons are active (high intracellular [Ca2+]). The ratio of red:green emission is stable such that it is quantifiable via subsequent microscopy. (B) Increased neural activity during TBI is represented by increased red:green emission (red pseudocoloured to magenta) in the hindbrain of larvae ( $\left.\mathbf{B}^{\prime \prime}\right)$, compared to larvae not receiving TBI ( $\mathbf{B}^{\prime}$ ) or fish not exposed to photoconverting light ("no PC" in panel B). These representative maximum intensity projection images show dorsal view of zebrafish brain (anterior at top), including merged, or red or green channels alone. (C) Heatmaps encode the CaMPARI signal (higher neural activity = higher red:green = hotter colours), highlighting location of increased neural activity during TBI relative to control larvae not receiving TBI. (D) Quantification of CaMPARI output in the hindbrain area reveals a significant increase in the neuronal excitability during TBI compared to control group not receiving TBI $\left({ }^{*} p<0.05\right.$. Each data point is an individual larva). 
625 subsequently exhibit a significantly higher propensity for spontaneous seizures.

\section{Traumatic brain injury on Tau biosensor zebrafish larvae induced GFP+ puncta}

628 After validating that our method was able to induce traumatic brain injury upon 629 zebrafish larvae, we next asked whether TBI induces tau aggregates in our tau

630 biosensor model. Initially, we evaluated if our TBI method would induce aggregation of 631 fluorescent proteins in models expressing GFP alone or other biosensor proteins such 632 as SOD1-GFP. Following TBI, and regardless of injury intensity, no GFP+ aggregates 633 were detected in these controls (Fig. S5 A-C). Similar results were obtained with other 634 transgenic zebrafish that express GFP in motor neurons (data not shown). Further, our 635 Tau4R-GFP fish additionally express an unmodified GFP variant in the active heart 636 muscle, and this robust GFP showed no sign of aggregation following TBI. Remarkably, 637 in these same individual Tau4R-GFP larvae we detected Tau4R-GFP biosensor GFP+ 638 puncta in both brains and spinal cords following TBI (Fig. 5A-B). The abundance of 639 GFP+ puncta increased with time following the injury (Fig 5C-D). To determine if the 640 severity of tauopathy varies coordinately with severity of the traumatic injury, we 641 assessed the impact of different masses. Although some variability is evident, a dose642 response relationship is apparent such that the $65 \mathrm{~g}, 100 \mathrm{~g}$ and $300 \mathrm{~g}$ weights induced 643 more tau aggregates compared to the control and $30 \mathrm{~g}$ weight (Fig. S8E). The heaviest 644 weight $(300 \mathrm{~g})$ induced significantly more tau aggregates versus the control group or the 645 group with the $30 \mathrm{~g}$ weight $(p<0.01$ and $p<0.001$, respectively). Therefore, we decided to 646 use both the 300 and $65 \mathrm{~g}$ weights for subsequent experiments. We evaluated whether 647 dropping the light weight once or multiple times would affect the number of tau 648 aggregates on the spinal cord as well as dropping the weight once on three consecutive 

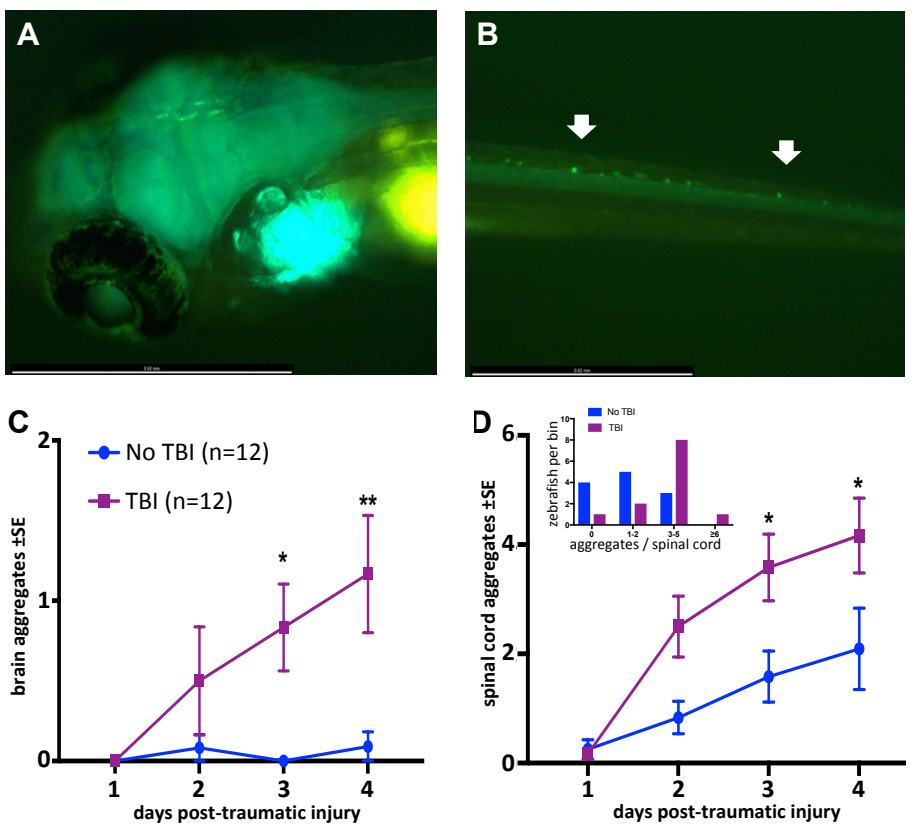

Fig 5. Traumatic brain injury (TBI) induces tauopathy in larval zebrafish. (A) GFP+ Tau puncta are detected in the brain of Tau4R-GFP biosensor zebrafish at 5 days post traumatic brain injury (dpti). (B) Tau aggregates formed on the spinal cord as a result of the traumatic brain injury as shown by arrows. (C) Tauopathy significantly increases over time following TBI compared to control group (No TBI) $\left({ }^{*} p<0.05\right.$ at $3 \mathrm{dpti}$ and $* * p<0.01$ at $4 \mathrm{dpti}$. (D) The number of tau aggregates in spinal cord significantly increases over time following TBI compared to control group ( ${ }^{*} p<0.05$ at $3 \mathrm{dpti}$ and 4dpti). Inset: Tau4R-GFP zebrafish larvae subjected to TBI develop more GFP+ puncta compared to the control group by $3 \mathrm{dpti}$ (inset plot is similar to Fig. 2E). (E-H) Post-traumatic
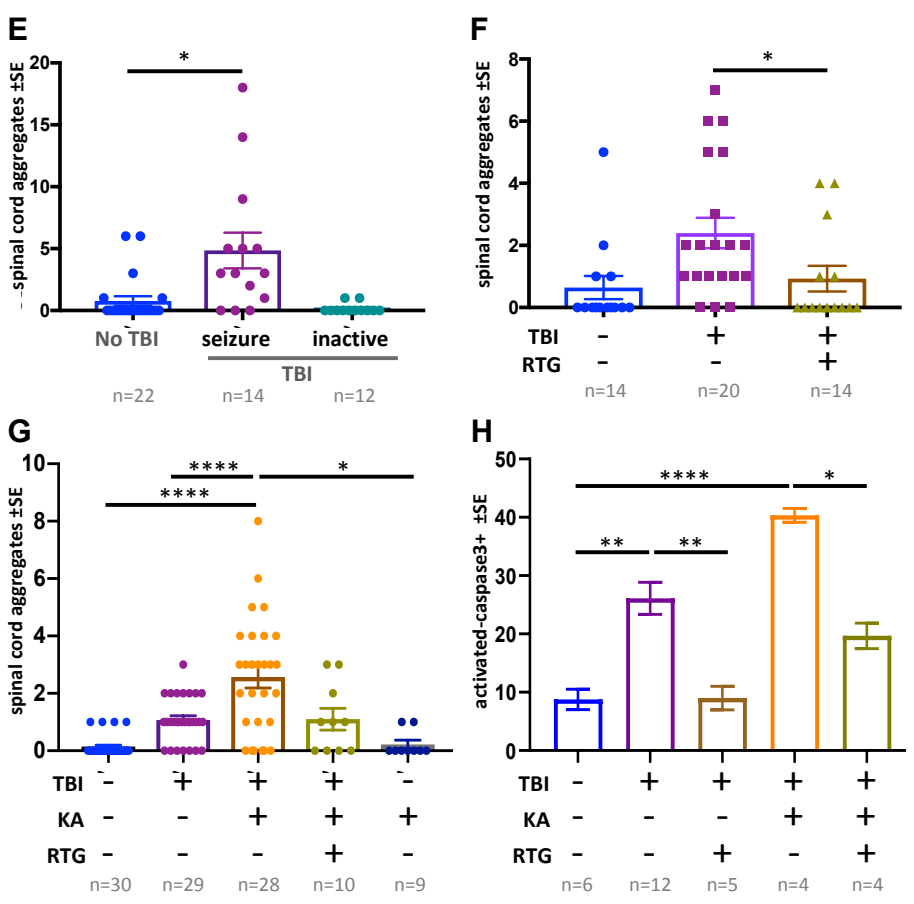

seizures link TBI to tauopathy. (E) Following TBI, larvae displaying post-traumatic seizures developed many more tau aggregates relative to those not displaying post-traumatic seizures $(* * * p<0.001)$. (F) Inhibiting post-traumatic seizures with the anti-convulsant retigabine (RTG, 10 $\mu M$ ) significantly decreased the abundance of GFP+ puncta in the spinal cord $(p<0.05)$. (G) Increasing post-traumatic seizure using the convulsant kainate $(\mathrm{KA}, 100 \mu \mathrm{M})$ significantly increased the formation of tau aggregation following TBI; this effect was prevented by co-treatment with anti-convulsant RTG. (H) Blunting post-traumatic seizures with RTG reduced TBI-related cell death. The main impact of RTG was specific to its anticonvulsant modulation of seizures because its effects were reversed by convulsant KA. Colour scheme in panel $\mathrm{C}$ applies to other panels. $\mathrm{n}=$ number of zebrafish larvae. 
650 days, perhaps reminiscent of repetitive sports injury. We observed an increase in the

651 abundance of tau aggregates when the weight was dropped multiple times during one

652 day, or over three consecutive days, but this increase was not statistically significant

653 (Fig. S8 B-D).

654 The GFP+ tau aggregates formed in the brain region following TBI tend to form 655 fused shapes (Fig. S8A) reminiscent of the spontaneous aggregates described above.

656 The aggregates on the spinal cord, however, had similar shapes to aggregates detected

657 post brain-injections, but with qualitatively less brightness in some instances. Multi-day

658 monitoring of individual larvae (Fig. S6 \& S7) revealed variation in formation of tau

659 aggregates amongst individual TBI larvae. We monitored the abundance of tau 660 aggregates within individual fish over time following TBI and found that the average 661 tauopathy significantly increased compared to the control group ( $p<0.05$ at $3 \mathrm{dpti}$ and $662 p<0.01$ at 4 dpti (days post traumatic injury) (Fig. 5C,D). Analysis of distribution of larvae 663 binned into the number of Tau4R-GFP+ puncta at 3 dpti showed that more larvae 664 developed Tau4R-GFP+ puncta compared to the control group (inset in Fig 5D). 665 Considering that many of the larvae subjected to TBI formed Tau4R-GFP+ puncta in the 666 brain that had a fused pattern (Fig. S8A), we focused on tau aggregates that formed on 667 the spinal cord as their abundance could be most efficiently quantified compared to 668 aggregates that formed in the brain.

\section{Post-traumatic seizure intensity influences tauopathy progression}

671 Considering the clinical prominence of post-traumatic seizures following TBI, and 672 the suggested role of cell stress and increased neural activity in promoting protein 
673 misfolding diseases (Kovacs et al., 2014; Sanchez et al., 2018), we speculated that 674 post-traumatic seizures might form a causal link between TBI and subsequent 675 tauopathy. We first asked if a correlation exists between seizure intensity and extent of 676 tauopathy. Following TBI, some larvae exhibited seizure-like movements, while some 677 did not seem to move abnormally relative to untreated fish (Fig. $3 \mathrm{H}$ ). We sorted the 678 larvae subjected to TBI into groups exhibiting the seizure-like behavior and those that 679 displayed no overtly abnormal movement. Larvae exhibiting seizure-like behavior after 680 TBI went on to develop abundant spinal cord aggregates (5-fold increase, $p<0.001)$ in 681 comparison to larvae that showed no seizure-like response to TBI (Fig. 5E).

682 To assess the hypothesis that seizure activity has a causal role in increasing the 683 abundance of tau aggregates in our TBI model, we employed convulsant and anti684 convulsant drugs to modulate the seizure intensity and in vivo neural activity. We 685 selected drugs that are well-established to behave similarly in zebrafish as in mammals, 686 though it is perhaps notable that the multi-day drug application used here is longer than 687 the acute applications typically considered in zebrafish (Ellis et al., 2012). Our 688 hypothesis predicted that decreasing seizure-like activity following TBI would reduce 689 tauopathy. Indeed, applying the anti-convulsant drug Retigabine (RTG), that opens 690 voltage-gated potassium channels (KCNQ, Kv7), resulted in a significant decrease in 691 the abundance of GFP+ puncta $(p<0.05)$ with many TBI larvae not developing any 692 Tau4R-GFP aggregates (Fig 5F).

693 Similarly, intensifying post-traumatic seizures via application of the convulsant 694 kainate increased the abundance on tauopathy 4-fold ( $p<0.001$. Fig $5 G$ ) in a dose695 dependent manner (Fig S9). Kainate did not increase Tau4R-GFP+ puncta in the 
696 absence of TBI. Surprisingly, the convulsant 4-aminopyridine did not increase tauopathy 697 (explored below).

698 To assess if the impacts of kainate and retigabine on tauopathy were directly due to 699 their modulation of post-traumatic seizures, we applied effective doses of each in 700 concert. Co-application of kainate and retigabine following TBI produced an abundance

701 of Tau4R-GFP+ puncta that was indistinguishable from larvae receiving TBI without 702 pharmacology (Fig 5G).

703 TBI-induced cell death was likewise correlated with the intensity of post-traumatic 704 seizures. Co-application of kainate and retigabine following TBI increased or decreased, 705 respectively, the abundance of cell death in a manner coordinate with the tauopathy 706 (Fig 5H).

707 Overall, convulsant and anti-convulsant drugs acted to increase and decrease TBI708 induced tauopathy, respectively. The drugs appear to be specific - their individual 709 impacts on tauopathy and cell death are largely attributable to their epileptic and anti710 epileptic modulation of post-traumatic seizures, because when kainate and retigabine 711 were applied concurrently they negated each others' effects.

\section{Appearance of tauopathy following TBI requires endocytosis}

714 To further examine increased seizure activity after TBI, we applied 4-aminopyridine 715 (4-AP), a $\mathrm{K}_{\mathrm{v}}$ channel blocker and convulsant drug. We predicted that raising the level of 716 seizure activity would elevate tauopathy abundance in our TBI model, aligning with our 717 observations following application of kainate (above). Surprisingly, higher doses of 4-AP 718 consistently abrogated the appearance of tau aggregates. Treating TBI larvae with 200 
719 or $800 \mu \mathrm{M}$ of $4-\mathrm{AP}$ for a prolonged period (38 hours, beginning 24 hours post traumatic 720 injury) significantly inhibited the abundance of Tau4R-GFP+ puncta in the TBI group 721 (Fig. 6A-B, Fig. S10A-B). Analysis of the distribution of larvae linked to the number of 722 tau aggregates supported this finding with no zebrafish larvae developing aggregates in 723 groups treated with 4-AP (Fig. S10C). It is worth noting that 4-AP is commonly used in 724 zebrafish models of epilepsy, but rarely used for prolonged treatment. To evaluate if the 725 time at which treatments are administered plays a role in this unexpected result, we 726 treated larvae with $200 \mu \mathrm{M}$ 4-AP at earlier time points, specifically during traumatic brain 727 injury and 1.5 hours later. We kept the duration of 4-AP treatment the same as previous 728 experiments (38 hours). We found that administering 4-AP during different time 729 windows relative to the traumatic brain injury did not measurably alter the inhibitory 730 action of 4-AP on the abundance of tau aggregates (Fig. S10D). A similar observation 731 was made when the duration of the 4-AP treatment was reduced to 24 hours (Fig. 732 S10E).

733 Next, we considered if this unexpected inhibition of tauopathy by high-dose 4-AP 734 convulsant is a direct consequence of increased neural activity (e.g. perhaps via neural 735 exhaustion). We found that larvae receiving TBI and 4-AP continued to exhibit a lack of 736 tau aggregates when co-treated with anti-convulsant retigabine $(p<0.0001)$ (Fig. 6B).

737 This suggested that high doses of 4-AP block the formation of tau aggregates via a 738 mechanism independent of its convulsant activity.

739 To resolve a mechanism whereby high doses of 4-AP reduced tau pathology, 740 contrary to our predictions above regarding neural hyperactivity, we considered 741 previous in vitro work that demonstrated high concentrations of 4-AP cause reduced 

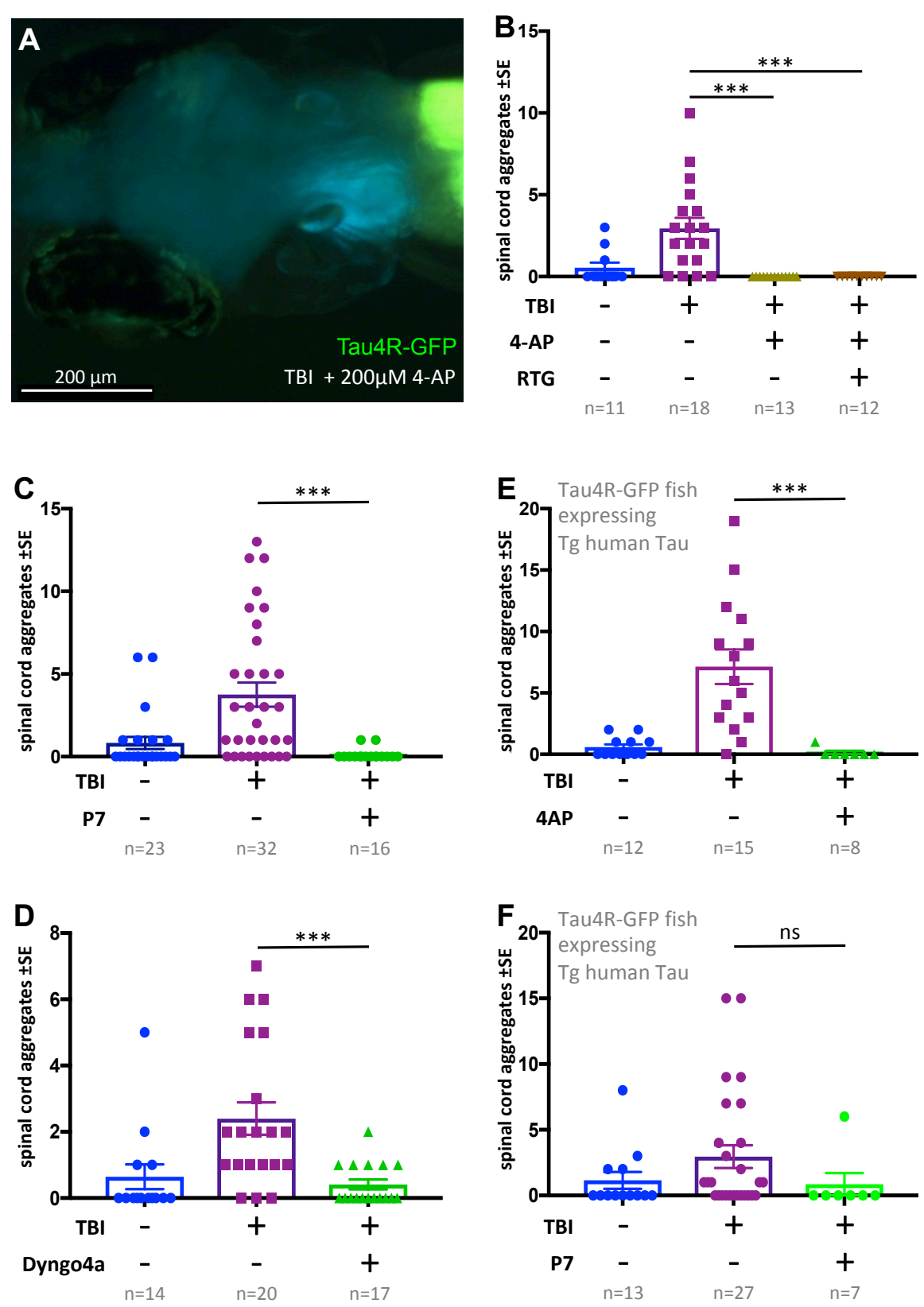

Fig 6. Tauopathy induced by traumatic brain injury (TBI) was attenuated by 4-aminopyridine (4-AP) via mechanisms independent of seizures. (A) Tau4R-GFP biosensor zebrafish larvae subjected to TBI and treated with the convulsant 4-AP show no brain puncta. (B) 4-AP significantly reduced (apparently eliminated) the abundance of GFP+ puncta in the brain and spinal cord compared to untreated TBI control. The impact of 4-AP on tauopathy appears to be independent of its actions on post-traumatic seizures because reducing the latter with anti-convulsant retigabine (RTG) had no measurable effect. See also Figure S10. C-F. Pharmacological inhibition of endocytosis reduced tauopathy following TBI. (C) Blocking endocytosis with Pyrimidyn-7 (P7) treatments significantly inhibited the formation of Tau4RGFP+ puncta following TBI in zebrafish larvae ( $\left.{ }^{* * *} p<0.001\right)$. (D) Dyngo 4a treatment significantly reduced Tau aggregates in the spinal cord $(* * * p<0.001)$ in a manner similar to P7. (E) 4-AP treatment significantly inhibited the formation of Tau4R-GFP+ puncta in the spinal cord $(* * * p<0.001)$ of Tau biosensor line that also express human Tau (ON4R) after traumatic brain injury compared to untreated TBI control group. (F) A notable reduction in Tau aggregates was observed in the same line after treatment with P7 drug. Statistical analysis shows no significance difference between groups. $n=$ number of larvae. 
743 endocytosis of synaptic vesicles (Cousin and Robinson, 2000). To examine if the

744 inhibitory actions of 4-AP on the abundance of tau aggregates in our TBI model is

745 consistent with an endocytosis inhibition mechanism, we treated our tau biosensor

746 larvae post traumatic injury with Pyrimidyn-7 (P7), a potent dynamin inhibitor that is

747 known to block endocytosis (McGeachie et al., 2013), and analyzed the propagation of

748 tau pathology by quantifying the number of tau inclusions. Owing to the potency of P7

749 and its impact on the survival of larvae, we treated the larvae with it for 24 hours at 3

$750 \mu \mathrm{M}$. Similar to the findings with 4-AP, P7 treatments significantly inhibited the formation

751 of Tau4R-GFP+ puncta in TBI larvae ( $p<0.001)$ (Fig. 6C). We assessed further the role

752 of endocytosis by employing another dynamin- inhibitor drug, Dyngo 4a, that is less

753 potent than P7 (McCluskey et al., 2013). We obtained similar results in which Dyngo 4a

754 treatments significantly reduced tauopathy in our TBI model (Fig. 6D). To determine if

755 these results are applicable to human tau, we induced traumatic brain injury on double-

756 transgenic larvae expressing both human tau (the ON4R human Tau isoform, see Bai et

757 al., 2007) and our tau biosensor reporter, followed by treatment with either 4-AP or P7.

758 Apart from the untreated control, both groups treated with 4-AP or P7 exhibited a

759 noticeable reduction in abundance of tau aggregates. While the decrease in the case of

760 P7 was not statistically significant, statistical analysis showed significance after 4-AP

761 treatments $(p<0.001)$ (Fig $6 \mathrm{E}$ and F). These findings confirmed the ability of 4-AP and

762 dynamin inhibitors of reducing human tau aggregates in our TBI larvae. 


\section{Discussion:}

The consequences of concussive blasts and TBI extend beyond the proximate 766 injury - they are prominent risk factors for devastating dementias including $A D, C T E$ 767 and other tauopathies. Identifying the causal links that entwine TBI with subsequent 768 tauopathies would inspire improved diagnostics and therapeutics. Investigating these 769 mechanisms has been hampered by lack of tractable models, since cell culture 770 platforms cannot faithfully represent the injury or the response-to-injury or the treatment 771 thereof. Indeed, TBI and tauopathies are complex tissue and systems-level events with 772 pathobiology progressing on a backdrop of dynamic vigorous neural function, prion-like 773 vectoring of misfolded proteins via glymphatic and blood vasculature, immune and 774 support cells, sleep physiology, homeostatic regulation and complex drug metabolism. 775 Rats have been the favoured animal model for TBI, and mice can complement this as 776 insightful models of tauopathy, yet both are challenged by expense, ethical 777 considerations, and CNS tissues that are relatively inaccessible to (longitudinal) 778 visualization of cellular events in living individuals (Bodnar et al., 2019; Marklund, 2016; 779 Meconi et al., 2018; Pham et al., 2019). Here, our zebrafish models imperfectly replicate 780 human TBI and tauopathy, but by addressing many of these challenges in an accessible 781 and vibrantly active vertebrate brain, we offer an innovative approach for the study of 782 prion-like events, tauopathy and/or TBI.

783 Here, we introduce a simple method for delivering TBI to larval zebrafish that can be 784 scaled to high-throughput and adopted at low expense. The tractability and 785 transparency of zebrafish larvae allowed us to deploy genetically encoded fluorescent 786 reporters that were validated to (i) uniquely quantify neural activity on freely behaving 
787 animals during TBI, and (ii) effectively document prion-like tauopathy in individual 788 subjects over multiple days. The accessibility of this platform to pharmacology allowed

789 us to query cell biology events in vivo and support a role for endocytosis in prion-like 790 progression and TBI-induced tauopathy. Further, anti-convulsant drugs were potent 791 mitigators of the tauopathy and cell death that emerged subsequent to TBI; these

792 effects were attributable to suppression of post-traumatic seizures (as proven by the 793 anticonvulsant's therapeutic effects being reversed by co-application of convulsant

794 drugs). It remains to be seen if this data has any bearing on the long-term clinical 795 management of TBI patients, and we newly speculate that prophylactic application of 796 anti-convulsants (already common for blunting of patient's post-traumatic seizures) 797 might hinder progression of tauopathies including CTE and AD. If true, then debates 798 regarding the optimal regimen of anti-epileptics for TBI patients should consider their 799 potential for providing long-term benefits on dementias.

800

801 Prion-like tauopathy induced by TBI

802 It is well established that TBI induces tauopathy, and over the past decade, 803 numerous in vitro and in vivo studies have supported that tau proteins possess prion804 like properties. Indeed seeding, templated misfolding (conversion) and spread to 805 synaptically connected regions has been documented in tauopathies such as $A D$ and 806 FTD (Ayers et al., 2018; de Calignon et al., 2012; Goedert et al., 2017a; Goedert et al., 807 2017b; Iba et al., 2015; Woerman et al., 2016). Various mechanisms have been 808 proposed for the transcellular transfer of tau seeds including release mechanisms via 809 exosomes, or cellular uptake mechanisms via endocytosis (Demaegd et al., 2018; 
810 Evans et al., 2018; Wang et al., 2017; Wu et al., 2013). Nonetheless, these suggested

811 mechanisms were postulated based on in vitro evidence as there is a lack of

812 appropriate models that can visualize and manipulate the prion-like spread of tau

813 pathology between tissues in a vibrant brain. It is unclear if these mechanisms are

814 universal in progression of all tauopathies or if there are factors and mechanisms that

815 are unique to each disease. Our zebrafish models allow us to study the progression and

816 spread of TBI-induced tauopathy longitudinally in living animals, an experimental

817 advantage that is unmatched among TBI animal models.

818 Regarding TBI, a large knowledge gap exists regarding how tau seeds are released

819 and/or internalized by adjacent (or far-flung) cells - indeed the prion-like properties of

820 tau species following TBI had not been assessed until very recently (Woerman et al.,

821 2016; Zanier et al., 2018). Moreover, the focus in the literature has mostly been directed

822 towards repetitive mild trauma as it is most associated with CTE, yet the various forms

823 of TBI all are considered risk factors for neurodegeneration. The recent revelation that

824 most TBI patients, whether they suffered from single or repetitive brain trauma, all

825 exhibited tau pathology similar to CTE (Washington et al., 2016; Zanier et al., 2018)

826 suggests all forms of TBI might incorporate tauopathies. Our mode of TBI on larval

827 zebrafish entails a pressure wave that most closely mimics a blast injury (e.g. as

828 experienced by military personnel or civilians near an explosion), but the etiology

829 leading to tauopathy probably has many similarities regardless of the mode of the

830 initiating TBI.

831

832 Post-traumatic seizures accelerate tauopathy following TBI 
We inspected the role of seizure activity and/or neuronal excitability, as well as the

834 role of dynamin-dependent endocytosis, during the progression of tauopathy after TBI.

835 We focussed our attention on seizure activity in part because seizures frequently occur

836 in TBI patients following blast traumatic injury (Englander et al., 2014; Kovacs et al.,

837 2014). We hypothesized that neuronal excitability and seizure activity after TBI can play

838 a role in accelerating the wide dissemination of tau pathology. As such, we introduced

839 two new approaches to test this hypothesis. The first approach was to engineer a novel

840 in vivo tau biosensor model in zebrafish that can visualize pathological tau spreading

841 and accumulation within the intact and vibrant CNS. The tau biosensor zebrafish

842 express human tau4R-GFP reporter protein, and we confirmed its ability to detect tau

843 seeds from various sources both in vivo and in vitro, similar to previously engineered in

844 vitro models (Kaufman et al., 2016; Sanders et al., 2014). Our second approach was to

845 introduce and optimize an elegantly simple technique to cause pressure-wave induced

$846 \mathrm{TBI}$, similar to human blast TBI, in zebrafish larvae. We endeavoured to inflict injury on

847 larval zebrafish rather than adults because of the synergistic advantages that larval

848 zebrafish provide: these include economical access to large numbers of individuals and

849 associated statistical power, and the tractability of larvae for high-throughput in vivo

850 screening of therapeutic agents (Saleem and Kannan, 2018). Larval zebrafish provide a

851 large economic advantage compared to adults, with respect to time, cost per individual

852 and space consumed in animal housing. Moreover, injuring animals in larval stages is

853 viewed as an ethically favourable Replacement [sensu "the three Rs" of (Russell and

854 Burch, 1959)] compared to injuring adult subjects. Thus, regardless of any bioethical

855 considerations based on taxonomy, larval fish (that are accessible early in their 
856 development via external fertilization of eggs) are ethically advantageous to rodents

857 (that are accessible for TBI only at postnatal stages) when considering highly invasive

858 procedures like TBI. The latter conclusion relies on the assumption that the knowledge

859 gained is of value, i.e. relevant to appreciating disease etiology.

860 Our data argue that our TBI methods are germane to clinical aetiology, because

861 (akin to existing animal models of TBI) we were able to confirm the presence of various

862 markers associated with brain injury, such as cell death, abnormalities in blood flow,

863 hemorrhage and the occurrence of post-traumatic seizures.

864 The post-traumatic seizures apparent in our TBI model led us to consider the neural

865 events occurring during the $\mathrm{TBI}$, and their potential bearing on the correlation between

866 neural activity and tauopathy. Few studies examine how TBI impacts neuronal circuits,

867 especially in vivo, and these typically consider events several hours or days after brain

868 trauma (Bugay et al., 2019). This may be of importance when considering evaluating

869 the reasons behind the developments of post-traumatic seizures and epilepsy. In a

870 controlled cortical impact model of TBI, an initial decrease or loss in neuronal activity is

871 recorded after injury before a rise in neuronal activity is noted (Ping and Jin, 2016).

872 Whether this occurs in different types of TBI, like blast TBI, was unexamined. To

873 address this, we performed TBI on larval zebrafish expressing CaMPARI, a genetically

874 encoded optogenetic reporter of neural activity. CaMPARI is particularly ideal for this

875 question, as its reportage of neural activity (a stable and quantifiable shift from green to

876 red fluorescence) occurs only during user-defined times and that reportage is relatively

877 permanent. This allowed us to quantify the CNS activity that had occurred during TBI,

878 by characterizing the ratio of red:green fluorescent emission using confocal microscopy 
879 after the TBI injury was completed. This approach therefor allows relatively easy access

880 to quantifying neural activity during injury in an unencumbered freely-swimming animal.

881 Here, we revealed for the first time a snapshot of neurons becoming active at the

882 moment of TBI. Our results demonstrated an increase in neuronal excitability upon TBI,

883 which may contribute to the frequency of post-traumatic seizures observed in our

884 model, other blast TBI models and TBI patients (Bugay et al., 2019). Regarding the

885 etiology of tauopathy subsequent to TBI, the CaMPARI quantification provided us

886 important validation that neural activity was substantively impacted by TBI,

887 complementing the evidence of increased seizure-like movements. This supported our

888 rationale that convulsant and anti-convulsant drug treatments might modulate neural

889 activity and thereby accelerate or decelerate tauopathy accumulation.

890 Indeed we had noted the occurrence of post-traumatic seizures in most of our TBI

891 samples, which is in agreement with the prevalence of seizures in blast TBI patients and

892 TBI rodent models (Bugay et al., 2019; Kovacs et al., 2014). However, whether post-

893 traumatic seizures contribute to prion-like spreading of tau pathology (observed after

894 TBI or not) was unknown. Beyond TBI, several investigations have supported an

895 association between tau pathology and seizures (Sanchez et al., 2018; Tai et al., 2016).

896 Studies on epileptic human temporal structure revealed accumulation of tau aggregates

897 (Sanchez et al., 2018). In 3XTg AD mice, induced chronic epilepsy was associated with

898 changes of inter-neuronal p-tau expression (Yan et al., 2012). Additionally, data

899 obtained from post-mortem analysis of patient tissues with $A D$ and drug resistant

900 epilepsy uncovered a correlation between symptomatic seizures, increased Braak 901 staging and accelerated tau accumulation (Thom et al., 2011). Interestingly, the 
902 presence of tau deposits in epileptic patients and the similarity of its pathology to CTE

903 suggest a conceivable role for seizures influencing the progression of tau pathology in a

904 similar manner to TBI (Puvenna et al., 2016). Indeed, our data from the application of

905 the convulsant kainate here support the role of post-traumatic seizure in enhancing tau

906 abundance and cell death in our TBI model (Fig. 5G,H). This finding is in line with

907 observations in a patient with epilepsy and a history of head injury, in which progressive

908 tau pathology was noted (Geddes et al., 1999; Thom et al., 2011). Intriguingly, reducing

909 seizure activity after TBI via anti-convulsant drugs was able to significantly reduce

910 tauopathy and cell death, providing further evidence of the relationship between

911 seizures and tauopathy in $\mathrm{TBI}$ (Fig $5 \mathrm{~F}, \mathrm{G}$ and $\mathrm{H}$ ). The mechanism of drug action

912 appears to be dominated by its anticonvulsant properties, because its effects were

913 reversed by co-application of convulsants. Thus, anti-convulsants are intriguing as a

914 route to slowing progression of tauopathy following TBI, and it is encouraging that they

915 are already commonly deployed to prevent post-traumatic seizures.

916

917 Endocytosis mediates prion-like spread of tauopathy

918 One particular convulsant drug, 4-AP, inhibited tauopathy in our TBI model (Fig 6 A

919 and B), contrary to our hypothesis that seizure intensity is positively correlated with

920 tauopathy following TBI. 4-AP is a voltage-gated potassium channel blocker that

921 enhances neuronal firing activity and has been used often in zebrafish seizure studies

922 (Kasatkina, 2016; Liu and Baraban, 2019; Lundh, 1978; R Kanyo, IN REVISION Jan

923 16th, 2020 ; Winter et al., 2017). Yet, 4-AP is rarely administered for prolonged

924 treatments such as those we deployed here, e.g. past studies rarely exceed one hour of 
925 4-AP (Winter et al., 2017). Thus, we considered that our high dose and prolonged

926 stimulation with 4-AP may have led to off-target effects; we confirmed this insomuch

927 that the 4-AP's inhibition of tauopathy was not related to its convulsant properties (as

928 determined by 4-AP's effects being unaltered by potent anti-convulsants (Fig. 6B)).

929 Indeed previous in vitro work revealed high concentrations or prolonged stimulation with

930 4-AP has off-target effects via inhibiting dynamin, which is important for the endocytosis

931 of synaptic vesicles at the nerve terminals (Cousin and Robinson, 2000). The inhibition

932 of endocytosis observed in that study was independent of 4-AP-dependent seizure 933 activity.

934 We further queried the potential role of dynamin-dependent endocytosis in the 935 prion-like progression of tau pathology after TBI by applying endocytosis inhibitors that 936 target dynamin. Dynamin is a GTPase involved in two mechanisms of endocytosis that 937 are important for synaptic vesicle transport (Singh et al., 2017). Empirical work on

938 human stem cell-derived neurons has indicated that tau aggregates are internalized via 939 dynamin-dependent endocytosis and that blocking other endocytosis pathways 940 independent of dynamin, such as bulk endocytosis and macropinocytosis, did not

941 disrupt tau uptake (Evans et al., 2018). On the contrary, inhibiting dynamin significantly 942 decreased the internalization of tau aggregates. Our results are in line with the 943 previously mentioned findings that show tau progression in TBI models depends on 944 dynamin-dependent endocytic pathways - blocking them with two different inhibitors 945 (and with 4-AP) dramatically lessened the abundance of tau seeds (Fig 6C-F). Hence, 946 our findings not only provide in vivo validation of past in vitro works, but also suggest 
947 mechanisms underlying prion-like spreading of tau seeds in TBI and CTE that could aid

948 in developing therapeutic strategies.

950 Limitations of our approach

951 Our tauopathy biosensor, human Tau4R-GFP, was deployed in vivo and uniquely 952 able to detect significant increases (and decreases) in the abundance of tau aggregates

953 following various insults and treatments, typically in a dose-dependent manner and in

954 harmony with expected trends. Considering this success, it remains intriguing that a 955 subset of larvae exhibit GFP+ tau puncta despite receiving no known tauopathy956 inducing insults. This suggests the larvae express the transgene at a level near to a 957 threshold for producing spontaneous aggregates. We performed selective breeding to 958 minimize these occurrences and tentatively believe, after too few generations, that 959 genetics of the fish is a factor - substantial genetic variation exists in zebrafish in-bred 960 lines (Balik-Meisner et al., 2018; Guryev et al., 2006). However, we acknowledge the 961 variation could be a minor technical artefact rather than biological. More optimistically, 962 this inter-individual variation and stochastic appearance of tauopathy, in a high963 throughput model, could be leveraged to newly appreciate aspects of spontaneous AD 964 or other non-familial tauopathies. Regardless, future work will also need to characterize 965 the biochemistry and biophysics of the human Tau inclusions in zebrafish compared to 966 patients or rodent models.

967 Regarding our TBI methods, further refinements may yet be able to improve 968 consistency of the injury and reduce the apparent variability between individuals. This 969 variability is real, but somewhat offset by the large sample sizes attainable: our TBI 
970 methods offer the potent advantages of zebrafish larvae with respect to genetic and 971 drug accessibility in high-throughput formats, while also retaining the critical in vivo

972 complexity required to investigate disease etiology and treatments. Further, it remains

973 to be established if the mechanisms we reveal are ubiquitous across the various forms

974 of TBI: our model fills a gap by supplying a rare 'closed head' TBI model (as opposed to

975 the majority of animal models that access the brain by removing skull elements prior to

976 brain injury, see exceptions by (Meconi et al., 2018; Mychasiuk et al., 2014). Our model

977 might be most relevant to brain trauma experienced by the human foetus (e.g. during 978 car collisions or domestic abuse), considering the developmental stage and aqueous

979 media. Further work is also needed to appreciate how the physics of our blast injury is 980 altered by occurring at a small scale (e.g. larval brain is $<500 \mu \mathrm{m}$ ). At this point we are 981 left to assume that the cellular and physiological aspects of TBI we consider here are 982 sufficiently similar across all classes of $\mathrm{TBI}$, and thus the knowledge gleaned may be 983 variably applicable.

984 Finally, we have chosen to restrict our analysis to study of larval fish. While this 985 offers many logistical and ethical advantages detailed above, it limits our study to acute 986 effects occurring over the course of several days. Conclusions from such work, once 987 refined and validated using the power of the in vivo zebrafish larva model, should be 988 tested in rodent models where it is equally time-consuming to assess the long-term 989 efficacy of treatments on these progressive late-onset dementias.

990

991 Conclusion 
992 Currently, no available treatments are applicable to all tauopathies, which remain as 993 devastating and inevitably fatal dementias. Zebrafish larvae, fostered by appropriate 994 innovations, now offer a potent complement both to rodent models of TBI and to cellular 995 models of tauopathy. Our engineered fish allowed us to reveal post-traumatic seizures 996 as a druggable mechanistic link between TBI and the prion-like progression of 997 tauopathy. Intriguingly, our conclusions have potential for translation to TBI clinics 998 where anti-convulsants are already in use as prophylactics for post-traumatic epilepsy, 999 though further work remains to address if they mitigate (the risk or severity of) later 1000 progression of CTE, AD or other tauopathies. 


\section{Acknowledgements:}

1003

1004

1005 samples, advice, and access to cell culture infrastructure. Gavin Neil and Jenna

1006 Bratvold contributed to SOD1-GFP cloning and zebrafish transgenesis via modifying a

1007 vector provided by Neil Cashman and Edward Pokrishevsky. Mark Loewen provided

1008 advice on hydraulic measures of pressure. Sue-Ann Mok, Satya Kar, Oksana

1009 Suchowersky, David Westaway and Brian Christie provided comments on an earlier

1010 version of the manuscript.

1011

1012 Funding to HA was from the Saudi Arabia Cultural Bureau and Majmaah University. RK 1013 was supported by SynAD postdoctoral fellowship funded via Alzheimer Society of 1014 Alberta and Northwest Territories through their Hope for Tomorrow program and the 1015 University Hospital Foundation. LFL received Studentships from Alberta Innovates and 1016 NSERC. MGD received Studentships from Alberta Innovates and CIHR. Operating 1017 funds to EB were from CurePSP 655-2018-06 and 468-08, US Department of Veterans 1018 Affairs BX003168, and NIH NS080881; The contents of this article do not represent the 1019 views of the United States government. Operating funds to HW were from Alberta 1020 Innovates and the Alzheimer Society of Alberta and Northwest Territories through the 1021 joint Alberta Alzheimer's Research Program (AARP 201700005). Operating funds to 1022 WTA were also from the joint AARP (201700018), and from anonymous donors; donors 1023 played no role in study design, prioritization or data interpretation, or decision to publish. 1024 


\section{Author Contributions:}

1026 HA preformed the experiments, collected and analyzed data, composed and wrote the 1027 manuscript. RK collected and analyzed data for CaMPARI experiments. LFL preformed 1028 kainate and some retigabine experiments, and characterized pressure kinetics. RK-J 1029 and HW obtained the electron microscope images. QB and EAB engineered and 1030 provided human Tau Tg zebrafish. MGD engineered SOD1:GFP Tg zebrafish. WTA 1031 was the supervisory author, was involved in concept formation, data analysis and 1032 interpretation, and edited the manuscript. All authors advised on editing the manuscript. 1033

1034 Competing Interests:

1035 The authors have no competing interests to declare. 
1038 Asikainen, I., Kaste, M., and Sarna, S. (1999). Early and late posttraumatic seizures in traumatic brain injury rehabilitation patients: brain injury factors causing late seizures and influence of seizures on long-term outcome. Epilepsia 40, 584-589.

Ayers, J.I., Giasson, B.I., and Borchelt, D.R. (2018). Prion-like Spreading in 1042 Tauopathies. Biol Psychiatry 83, 337-346.

1043 Bai, Q., Garver, J.A., Hukriede, N.A., and Burton, E.A. (2007). Generation of a 1044 transgenic zebrafish model of Tauopathy using a novel promoter element derived from 1045 the zebrafish eno2 gene. Nucleic Acids Res 35, 6501-6516.

1046 Balik-Meisner, M., Truong, L., Scholl, E.H., Tanguay, R.L., and Reif, D.M. (2018). 1047 Population genetic diversity in zebrafish lines. Mamm Genome 29, 90-100.

1048 Bir, C., Vandevord, P., Shen, Y., Raza, W., and Haacke, E.M. (2012). Effects of variable 1049 blast pressures on blood flow and oxygen saturation in rat brain as evidenced using MRI. Magn Reson Imaging 30, 527-534.

Bodnar, C.N., Roberts, K.N., Higgins, E.K., and Bachstetter, A.D. (2019). A Systematic Review of Closed Head Injury Models of Mild Traumatic Brain Injury in Mice and Rats. J Neurotrauma 36, 1683-1706.

1054 Bugay, V., Bozdemir, E., Vigil, F.A., Holstein, D.M., Chun, S.H., Elliot, W., Sprague, C., 1055 Cavazos, J.E., Zamora, D.O., Rule, G., et al. (2019). A mouse model of repetitive blast traumatic brain injury reveals post-trauma seizures and increased neuronal excitability. J Neurotrauma.

Chauhan, N.B. (2014). Chronic neurodegenerative consequences of traumatic brain injury. Restor Neurol Neurosci 32, 337-365.

Chiti, F., and Dobson, C.M. (2006). Protein misfolding, functional amyloid, and human disease. Annu Rev Biochem 75, 333-366.

1062 Clavaguera, F., Akatsu, H., Fraser, G., Crowther, R.A., Frank, S., Hench, J., Probst, A., 1063 Winkler, D.T., Reichwald, J., Staufenbiel, M., et al. (2013). Brain homogenates from 1064 human tauopathies induce tau inclusions in mouse brain. Proc Natl Acad Sci U S A 110, 1065 9535-9540.

1066 Colin, M., Dujardin, S., Schraen-Maschke, S., Meno-Tetang, G., Duyckaerts, C., 1067 Courade, J.P., and Buee, L. (2020). From the prion-like propagation hypothesis to 1068 therapeutic strategies of anti-tau immunotherapy. Acta Neuropathol 139, 3-25.

1069 Cousin, M.A., and Robinson, P.J. (2000). Ca(2+) influx inhibits dynamin and arrests 1070 synaptic vesicle endocytosis at the active zone. J Neurosci 20, 949-957. 
1071 Cruz-Haces, M., Tang, J., Acosta, G., Fernandez, J., and Shi, R. (2017). Pathological 1072 correlations between traumatic brain injury and chronic neurodegenerative diseases.

1073 Transl Neurodegener 6, 20.

1074 de Calignon, A., Polydoro, M., Suarez-Calvet, M., William, C., Adamowicz, D.H., 1075 Kopeikina, K.J., Pitstick, R., Sahara, N., Ashe, K.H., Carlson, G.A., et al. (2012). 1076 Propagation of tau pathology in a model of early Alzheimer's disease. Neuron 73, 6851077697.

1078 Demaegd, K., Schymkowitz, J., and Rousseau, F. (2018). Transcellular Spreading of 1079 Tau in Tauopathies. Chembiochem 19, 2424-2432.

1080 DuVal, M.G., Oel, A.P., and Allison, W.T. (2014). gdf6a Is Required for Cone 1081 Photoreceptor Subtype Differentiation and for the Actions of tbx2b in Determining Rod 1082 Versus Cone Photoreceptor Fate. Plos One 9.

1083 Ellis, L.D., Seibert, J., and Soanes, K.H. (2012). Distinct models of induced hyperactivity 1084 in zebrafish larvae. Brain Res 1449, 46-59.

1085 Englander, J., Cifu, D.X., and Diaz-Arrastia, R. (2014). Information/education page. 1086

1094 Fisher, S., Grice, E.A., Vinton, R.M., Bessling, S.L., Urasaki, A., Kawakami, K., and

1105 Goedert, M., Eisenberg, D.S., and Crowther, R.A. (2017a). Propagation of Tau
1106 Aggregates and Neurodegeneration. Annu Rev Neurosci 40, 189-210. 1106 Tol2 transposon-mediated transgenesis in zebrafish. Nat Protoc 1, 1297-1305.

Fosque, B.F., Sun, Y., Dana, H., Yang, C.T., Ohyama, T., Tadross, M.R., Patel, R., Zlatic, M., Kim, D.S., Ahrens, M.B., et al. (2015). Neural circuits. Labeling of active neural circuits in vivo with designed calcium integrators. Science 347, 755-760.

Gardner, R.C., and Yaffe, K. (2015). Epidemiology of mild traumatic brain injury and neurodegenerative disease. Mol Cell Neurosci 66, 75-80.

Geddes, J.F., Vowles, G.H., Nicoll, J.A., and Revesz, T. (1999). Neuronal cytoskeletal changes are an early consequence of repetitive head injury. Acta Neuropathol 98, 171178. 
1107 Goedert, M., Masuda-Suzukake, M., and Falcon, B. (2017b). Like prions: the 1108 propagation of aggregated tau and alpha-synuclein in neurodegeneration. Brain 140, 1109 266-278.

1110 Goedert, M., Spillantini, M.G., Jakes, R., Rutherford, D., and Crowther, R.A. (1989). 1111 Multiple isoforms of human microtubule-associated protein tau: sequences and 1112 localization in neurofibrillary tangles of Alzheimer's disease. Neuron 3, 519-526.

1113 Guo, J.L., and Lee, V.M. (2011). Seeding of normal Tau by pathological Tau conformers 1114 drives pathogenesis of Alzheimer-like tangles. J Biol Chem 286, 15317-15331.

1115 Guo, J.L., Narasimhan, S., Changolkar, L., He, Z., Stieber, A., Zhang, B., Gathagan, 1116 R.J., Iba, M., McBride, J.D., Trojanowski, J.Q., and Lee, V.M. (2016). Unique 1117 pathological tau conformers from Alzheimer's brains transmit tau pathology in 1118 nontransgenic mice. J Exp Med 213, 2635-2654.

1119 Guryev, V., Koudijs, M.J., Berezikov, E., Johnson, S.L., Plasterk, R.H., van Eeden, F.J., 1120 and Cuppen, E. (2006). Genetic variation in the zebrafish. Genome Res 16, 491-497.

1121 Gutzman, J.H., and Sive, H. (2009). Zebrafish brain ventricle injection. J Vis Exp.

1122 Hanwell, D., Hutchinson, S.A., Collymore, C., Bruce, A.E., Louis, R., Ghalami, A., 1123 Allison, W.T., Ekker, M., Eames, B.F., Childs, S., et al. (2016). Restrictions on the 1124 Importation of Zebrafish into Canada Associated with Spring Viremia of Carp Virus. 1125 Zebrafish 13 Suppl 1, S153-163.

1126 Hay, J., Johnson, V.E., Smith, D.H., and Stewart, W. (2016). Chronic Traumatic 1127 Encephalopathy: The Neuropathological Legacy of Traumatic Brain Injury. Annu Rev 1128 Pathol 11, 21-45.

1129 Iba, M., Guo, J.L., McBride, J.D., Zhang, B., Trojanowski, J.Q., and Lee, V.M. (2013). 1130 Synthetic tau fibrils mediate transmission of neurofibrillary tangles in a transgenic 1131 mouse model of Alzheimer's-like tauopathy. J Neurosci 33, 1024-1037.

1132 Iba, M., McBride, J.D., Guo, J.L., Zhang, B., Trojanowski, J.Q., and Lee, V.M. (2015). 1133 Tau pathology spread in PS19 tau transgenic mice following locus coeruleus (LC) 1134 injections of synthetic tau fibrils is determined by the LC's afferent and efferent 1135 connections. Acta Neuropathol 130, 349-362.

1136 Johnson, V.E., Stewart, J.E., Begbie, F.D., Trojanowski, J.Q., Smith, D.H., and Stewart, 1137 W. (2013). Inflammation and white matter degeneration persist for years after a single 1138 traumatic brain injury. Brain 136, 28-42.

1139 Johnson, V.E., Stewart, W., and Smith, D.H. (2012). Widespread tau and amyloid-beta 1140 pathology many years after a single traumatic brain injury in humans. Brain Pathol 22, 1141 142-149. 
1142 Kanyo, R., Leighton, P.L.A., Neil, G.J., Locskai, L.F., and Allison, W.T. (2020). Amyloid1143 beta precursor protein mutant zebrafish exhibit seizure susceptibility that depends on 1144 prion protein. Exp Neurol, 113283.

1145 Kanyo, R., Wang, C.K., Locskai, L.F., Li, J., Allison, W.T., and H Kurata (IN REVISION 1146 as of Jan $16^{\text {th }}, 2020$ ). Functional and behavioral signatures of Kv7 activator drug subtypes. Epilepsia. ms\# EPI-00953-2019.

1150 Kaufman, S.K., Sanders, D.W., Thomas, T.L., Ruchinskas, A.J., Vaquer-Alicea, J.,

Kasatkina, L.A. (2016). 4-capital A, Cyrillicminopyridine sequesters intracellular $\mathrm{Ca}(2+)$ which triggers exocytosis in excitable and non-excitable cells. Sci Rep 6, 34749.

1154 Kovacs, G.G. (2017). Tauopathies. Handb Clin Neurol 145, 355-368.

1155 Kovacs, S.K., Leonessa, F., and Ling, G.S. (2014). Blast TBI Models, Neuropathology, 1156 and Implications for Seizure Risk. Front Neurol 5, 47.

1157 Kwan, K.M., Fujimoto, E., Grabher, C., Mangum, B.D., Hardy, M.E., Campbell, D.S., 1158 Parant, J.M., Yost, H.J., Kanki, J.P., and Chien, C.B. (2007). The Tol2kit: a multisite 1159 gateway-based construction kit for Tol2 transposon transgenesis constructs. Dev Dyn 1160 236, 3088-3099.

1161 Leighton, P.L.A., Kanyo, R., Neil, G.J., Pollock, N.M., and Allison, W.T. (2018). Prion 1162 gene paralogs are dispensable for early zebrafish development and have nonadditive 1163 roles in seizure susceptibility. J Biol Chem 293, 12576-12592.

1164 Lim, J., and Yue, Z. (2015). Neuronal aggregates: formation, clearance, and spreading. 1165 Dev Cell 32, 491-501.

1166 Liu, J., and Baraban, S.C. (2019). Network Properties Revealed during Multi-Scale 1167 Calcium Imaging of Seizure Activity in Zebrafish. eNeuro 6.

1168 Lucke-Wold, B.P., Nguyen, L., Turner, R.C., Logsdon, A.F., Chen, Y.W., Smith, K.E., Huber, J.D., Matsumoto, R., Rosen, C.L., Tucker, E.S., and Richter, E. (2015). Traumatic brain injury and epilepsy: Underlying mechanisms leading to seizure. Seizure 33, 13-23.

1172 Lundh, H. (1978). Effects of 4-aminopyridine on neuromuscular transmission. Brain Res 1173 153, 307-318.

1174 Maheras, A.L., Dix, B., Carmo, O.M.S., Young, A.E., Gill, V.N., Sun, J.L., Booker, A.R., 1175 Thomason, H.A., Ibrahim, A.E., Stanislaw, L., et al. (2018). Genetic Pathways of 1176 Neuroregeneration in a Novel Mild Traumatic Brain Injury Model in Adult Zebrafish. 1177 eNeuro 5. 
1178 Marklund, N. (2016). Rodent Models of Traumatic Brain Injury: Methods and 1179 Challenges. Methods Mol Biol 1462, 29-46.

1180 McCluskey, A., Daniel, J.A., Hadzic, G., Chau, N., Clayton, E.L., Mariana, A., Whiting, 1181 A., Gorgani, N.N., Lloyd, J., Quan, A., et al. (2013). Building a better dynasore: the 1182 dyngo compounds potently inhibit dynamin and endocytosis. Traffic 14, 1272-1289.

1183 McCutcheon, V., Park, E., Liu, E., Sobhebidari, P., Tavakkoli, J., Wen, X.Y., and Baker, 1184 A.J. (2017). A Novel Model of Traumatic Brain Injury in Adult Zebrafish Demonstrates 1185 Response to Injury and Treatment Comparable with Mammalian Models. J 1186 Neurotrauma 34, 1382-1393.

1187 McGeachie, A.B., Odell, L.R., Quan, A., Daniel, J.A., Chau, N., Hill, T.A., Gorgani, N.N., 1188 Keating, D.J., Cousin, M.A., van Dam, E.M., et al. (2013). Pyrimidyn compounds: dualaction small molecule pyrimidine-based dynamin inhibitors. ACS Chem Biol 8, 15071518.

McKee, A.C., Stein, T.D., Kiernan, P.T., and Alvarez, V.E. (2015). The neuropathology of chronic traumatic encephalopathy. Brain Pathol 25, 350-364.

Meconi, A., Wortman, R.C., Wright, D.K., Neale, K.J., Clarkson, M., Shultz, S.R., and Christie, B.R. (2018). Repeated mild traumatic brain injury can cause acute neurologic impairment without overt structural damage in juvenile rats. PLoS One 13, e0197187.

Mudher, A., Colin, M., Dujardin, S., Medina, M., Dewachter, I., Alavi Naini, S.M., Mandelkow, E.M., Mandelkow, E., Buee, L., Goedert, M., and Brion, J.P. (2017). What is the evidence that tau pathology spreads through prion-like propagation? Acta Neuropathol Commun 5, 99.

Murakami, T., Paitel, E., Kawarabayashi, T., Ikeda, M., Chishti, M.A., Janus, C., Matsubara, E., Sasaki, A., Kawarai, T., Phinney, A.L., et al. (2006). Cortical neuronal and glial pathology in TgTauP301L transgenic mice: neuronal degeneration, memory disturbance, and phenotypic variation. Am J Pathol 169, 1365-1375.

Mychasiuk, R., Farran, A., Angoa-Perez, M., Briggs, D., Kuhn, D., and Esser, M.J. (2014). A novel model of mild traumatic brain injury for juvenile rats. J Vis Exp.

Nakagawa, A., Manley, G.T., Gean, A.D., Ohtani, K., Armonda, R., Tsukamoto, A., Yamamoto, H., Takayama, K., and Tominaga, T. (2011). Mechanisms of primary blastinduced traumatic brain injury: insights from shock-wave research. J Neurotrauma 28, $1101-1119$.

Narasimhan, S., Guo, J.L., Changolkar, L., Stieber, A., McBride, J.D., Silva, L.V., He, Z., Zhang, B., Gathagan, R.J., Trojanowski, J.Q., and Lee, V.M.Y. (2017). Pathological Tau Strains from Human Brains Recapitulate the Diversity of Tauopathies in Nontransgenic Mouse Brain. J Neurosci 37, 11406-11423. 
1214 Nguyen, R., Fiest, K.M., McChesney, J., Kwon, C.S., Jette, N., Frolkis, A.D., Atta, C., 1215 Mah, S., Dhaliwal, H., Reid, A., et al. (2016). The International Incidence of Traumatic 1216 Brain Injury: A Systematic Review and Meta-Analysis. Can J Neurol Sci 43, 774-785.

1217 Ojo, J.O., Mouzon, B., Algamal, M., Leary, P., Lynch, C., Abdullah, L., Evans, J., 1218 Mullan, M., Bachmeier, C., Stewart, W., and Crawford, F. (2016). Chronic Repetitive Mild Traumatic Brain Injury Results in Reduced Cerebral Blood Flow, Axonal Injury, Gliosis, and Increased T-Tau and Tau Oligomers. J Neuropathol Exp Neurol 75, 636655.

Orr, M.E., Sullivan, A.C., and Frost, B. (2017). A Brief Overview of Tauopathy: Causes, Consequences, and Therapeutic Strategies. Trends Pharmacol Sci 38, 637-648.

1224 Peeraer, E., Bottelbergs, A., Van Kolen, K., Stancu, I.C., Vasconcelos, B., Mahieu, M., Duytschaever, H., Ver Donck, L., Torremans, A., Sluydts, E., et al. (2015). Intracerebral injection of preformed synthetic tau fibrils initiates widespread tauopathy and neuronal loss in the brains of tau transgenic mice. Neurobiol Dis 73, 83-95.

Pham, L., Shultz, S.R., Kim, H.A., Brady, R.D., Wortman, R.C., Genders, S.G., Hale, M.W., O'Shea, R.D., Djouma, E., van den Buuse, M., et al. (2019). Mild Closed-Head Injury in Conscious Rats Causes Transient Neurobehavioral and Glial Disturbances: A Novel Experimental Model of Concussion. J Neurotrauma 36, 2260-2271.

Pickett, E.K., Henstridge, C.M., Allison, E., Pitstick, R., Pooler, A., Wegmann, S., Carlson, G., Hyman, B.T., and Spires-Jones, T.L. (2017). Spread of tau down neural circuits precedes synapse and neuronal loss in the rTgTauEC mouse model of early Alzheimer's disease. Synapse 71.

1236 Ping, X., and Jin, X. (2016). Transition from Initial Hypoactivity to Hyperactivity in 1237 Cortical Layer V Pyramidal Neurons after Traumatic Brain Injury In Vivo. J Neurotrauma 1238 33, 354-361.

1239 Pokrishevsky, E., McAlary, L., Farrawell, N.E., Zhao, B., Sher, M., Yerbury, J.J., and Cashman, N.R. (2018). Tryptophan 32-mediated SOD1 aggregation is attenuated by pyrimidine-like compounds in living cells. Sci Rep 8, 15590.

1242 Puvenna, V., Engeler, M., Banjara, M., Brennan, C., Schreiber, P., Dadas, A., Bahrami, 1243 A., Solanki, J., Bandyopadhyay, A., Morris, J.K., et al. (2016). Is phosphorylated tau 1244

1246 Rimel, R.W., Giordani, B., Barth, J.T., Boll, T.J., and Jane, J.A. (1981). Disability caused 1247 by minor head injury. Neurosurgery $9,221-228$.

1248 Russell, W.M.S., and Burch, R.L. (1959). The principles of humane experimental 1249 technique (London,: Methuen). 
1250 Safar, J., Wille, H., Itri, V., Groth, D., Serban, H., Torchia, M., Cohen, F.E., and 1251 Prusiner, S.B. (1998). Eight prion strains have $\operatorname{PrP}(\mathrm{Sc})$ molecules with different 1252 conformations. Nat Med 4, 1157-1165.

1253 Saleem, S., and Kannan, R.R. (2018). Zebrafish: an emerging real-time model system 1254 to study Alzheimer's disease and neurospecific drug discovery. Cell Death Discov 4, 45.

1255 Salinsky, M., Storzbach, D., Goy, E., and Evrard, C. (2015). Traumatic brain injury and 1256 psychogenic seizures in veterans. J Head Trauma Rehabil 30, E65-70.

Sanchez, M.P., Garcia-Cabrero, A.M., Sanchez-Elexpuru, G., Burgos, D.F., and 1259 Serratosa, J.M. (2018). Tau-Induced Pathology in Epilepsy and Dementia: Notions from Patients and Animal Models. Int J Mol Sci 19.

1260 Sanders, D.W., Kaufman, S.K., DeVos, S.L., Sharma, A.M., Mirbaha, H., Li, A., Barker, 1261 S.J., Foley, A.C., Thorpe, J.R., Serpell, L.C., et al. (2014). Distinct tau prion strains 1262 propagate in cells and mice and define different tauopathies. Neuron 82, 1271-1288.

Singh, M., Jadhav, H.R., and Bhatt, T. (2017). Dynamin Functions and Ligands: Classical Mechanisms Behind. Mol Pharmacol 91, 123-134.

Stafstrom, C.E., and Carmant, L. (2015). Seizures and epilepsy: an overview for neuroscientists. Cold Spring Harb Perspect Med 5.

1267 Tai, X.Y., Koepp, M., Duncan, J.S., Fox, N., Thompson, P., Baxendale, S., Liu, J.Y., 1268 Reeves, C., Michalak, Z., and Thom, M. (2016). Hyperphosphorylated tau in patients with refractory epilepsy correlates with cognitive decline: a study of temporal lobe resections. Brain 139, 2441-2455.

1278 Uryu, K., Chen, X.H., Martinez, D., Browne, K.D., Johnson, V.E., Graham, D.I., Lee, 1279 V.M., Trojanowski, J.Q., and Smith, D.H. (2007). Multiple proteins implicated in 1280 neurodegenerative diseases accumulate in axons after brain trauma in humans. Exp 1281 Neurol 208, 185-192.

1282 Verweij, F.J., Revenu, C., Arras, G., Dingli, F., Loew, D., Pegtel, D.M., Follain, G., Allio, 1283 G., Goetz, J.G., Zimmermann, P., et al. (2019). Live Tracking of Inter-organ 1284 Communication by Endogenous Exosomes In Vivo. Dev Cell 48, 573-589 e574. 
Wang, Y., Balaji, V., Kaniyappan, S., Kruger, L., Irsen, S., Tepper, K., Chandupatla, R., Maetzler, W., Schneider, A., Mandelkow, E., and Mandelkow, E.M. (2017). The release and trans-synaptic transmission of Tau via exosomes. Mol Neurodegener 12, 5.

Washington, P.M., Villapol, S., and Burns, M.P. (2016). Polypathology and dementia after brain trauma: Does brain injury trigger distinct neurodegenerative diseases, or should they be classified together as traumatic encephalopathy? Exp Neurol 275 Pt 3, 381-388.

1292 Westerfield, M. (2000). The zebrafish book. A guide for the laboratory use of zebrafish 1293 (Danio rerio), 4th edn (University of Oregon press, Eugene).

1294

1295

1296

1297

1298

1299

1300

1301

1302

1303

1304

1305

1306

1307

1308

1309

1310

1311

1312

1313

1314

1315

1316

1317

1318

1319

1320

White, R.M., Sessa, A., Burke, C., Bowman, T., LeBlanc, J., Ceol, C., Bourque, C., Dovey, M., Goessling, W., Burns, C.E., and Zon, L.I. (2008). Transparent adult zebrafish as a tool for in vivo transplantation analysis. Cell Stem Cell 2, 183-189.

Winter, M.J., Windell, D., Metz, J., Matthews, P., Pinion, J., Brown, J.T., Hetheridge, M.J., Ball, J.S., Owen, S.F., Redfern, W.S., et al. (2017). 4-dimensional functional profiling in the convulsant-treated larval zebrafish brain. Sci Rep 7, 6581.

Woerman, A.L., Aoyagi, A., Patel, S., Kazmi, S.A., Lobach, I., Grinberg, L.T., McKee, A.C., Seeley, W.W., Olson, S.H., and Prusiner, S.B. (2016). Tau prions from Alzheimer's disease and chronic traumatic encephalopathy patients propagate in cultured cells. Proc Natl Acad Sci U S A 113, E8187-E8196.

Wu, J.W., Herman, M., Liu, L., Simoes, S., Acker, C.M., Figueroa, H., Steinberg, J.I., Margittai, M., Kayed, R., Zurzolo, C., et al. (2013). Small misfolded Tau species are internalized via bulk endocytosis and anterogradely and retrogradely transported in neurons. J Biol Chem 288, 1856-1870.

Wu, J.W., Hussaini, S.A., Bastille, I.M., Rodriguez, G.A., Mrejeru, A., Rilett, K., Sanders, D.W., Cook, C., Fu, H., Boonen, R.A., et al. (2016). Neuronal activity enhances tau propagation and tau pathology in vivo. Nat Neurosci 19, 1085-1092.

Yamada, K., Holth, J.K., Liao, F., Stewart, F.R., Mahan, T.E., Jiang, H., Cirrito, J.R., Patel, T.K., Hochgrafe, K., Mandelkow, E.M., and Holtzman, D.M. (2014). Neuronal activity regulates extracellular tau in vivo. J Exp Med 211, 387-393.

Yan, X.X., Cai, Y., Shelton, J., Deng, S.H., Luo, X.G., Oddo, S., Laferla, F.M., Cai, H., Rose, G.M., and Patrylo, P.R. (2012). Chronic temporal lobe epilepsy is associated with enhanced Alzheimer-like neuropathology in 3xTg-AD mice. Plos One 7, e48782.

Zanier, E.R., Bertani, I., Sammali, E., Pischiutta, F., Chiaravalloti, M.A., Vegliante, G., Masone, A., Corbelli, A., Smith, D.H., Menon, D.K., et al. (2018). Induction of a transmissible tau pathology by traumatic brain injury. Brain 141, 2685-2699. 